\title{
Acceleration of Particles to High Energies in Earth's Radiation Belts
}

\author{
R.M. Millan • D.N. Baker
}

Received: 16 April 2012 / Accepted: 30 September 2012 / Published online: 25 October 2012

(C) The Author(s) 2012. This article is published with open access at Springerlink.com

\begin{abstract}
Discovered in 1958, Earth's radiation belts persist in being mysterious and unpredictable. This highly dynamic region of near-Earth space provides an important natural laboratory for studying the physics of particle acceleration. Despite the proximity of the radiation belts to Earth, many questions remain about the mechanisms responsible for rapidly energizing particles to relativistic energies there. The importance of understanding the radiation belts continues to grow as society becomes increasingly dependent on spacecraft for navigation, weather forecasting, and more. We review the historical underpinning and observational basis for our current understanding of particle acceleration in the radiation belts.
\end{abstract}

Keywords Particle acceleration · Radiation belts · Magnetosphere

\section{Introduction}

\subsection{Motivation}

Shortly after the discovery of Earth's radiation belts, the suggestion was put forward that processes occurring locally, in near-Earth space, might be responsible for the high energy particles observed there. Efforts were also carried out to search for an external source that could inject multi-MeV electrons into Earth's inner magnetosphere where they could then be trapped by the magnetic field. Energetic electrons are in fact observed in interplanetary space, originating at both Jupiter and the sun. However, the electron intensity in Earth's radiation belts is not correlated with the interplanetary intensity, and a significant external

R.M. Millan ( $₫)$

Department of Physics and Astronomy, Dartmouth College, 6127 Wilder Lab, Hanover, NH 03755, USA

e-mail: Robyn.Millan@dartmouth.edu

D.N. Baker

Laboratory for Atmospheric and Space Physics, and Department of Physics, and Department of Astrophysical and Planetary Sciences, University of Colorado, Boulder, CO 80309, USA 
source for radiation belt electrons has not been identified. All evidence supports the idea that Earth is a powerful accelerator of particles. We now know that Earth is even more efficient at accelerating particles than previously thought; observations indicate that this region of space is regularly depleted and replenished of relativistic electrons on timescales as short as a few hours.

How are electrons rapidly accelerated to relativistic energies in a seemingly benign environment such as Earth? Many of the acceleration mechanisms that are currently being studied in detail were actually proposed decades ago. However, our knowledge of the interplay between these mechanisms has been limited by the small number of observations made with modern instrumentation. Processes proposed on theoretical grounds have not been adequately tested with data. Because of the harsh radiation environment, spacecraft missions to the radiation belts are technically difficult, thus the radiation belts have been under-explored since their discovery in 1958. In August 2012, NASA launched the first spacecraft mission to the heart of the radiation belts in over 20 years. The Radiation Belt Storm Probes (RBSP) mission includes two identical spacecraft, heavily instrumented with state-of-the-art particle and field instruments, to provide multi-point measurements in the radiation belts (Ukhorskiy et al. 2011b). Other missions are currently being planned by Japan and Europe.

Today, we are in a position to take on new challenges to resolve the long-standing mysteries of the radiation belts. Strong variations in Earth's magnetic field have a significant effect on particle dynamics which can now be investigated with test-particle simulations on modern computers. The recent discovery of very large-amplitude whistler waves in the magnetosphere raises questions about the validity of the quasi-linear treatment of wave-particle interactions in the radiation belts. Over the next decade, radiation belt missions will provide detailed measurements of plasma waves and particle distributions, and theoretical tools will provide the interpretation needed to advance our understanding of particle acceleration occurring right on our doorstep.

The purpose of this review is to provide an overview of particle acceleration in Earth's radiation belts, with an emphasis on the observational characteristics of this region. Recent review papers have given extensive overviews of radiation belt modeling (Shprits et al. 2008a, 2008b), the solar wind influence on the radiation belts and different acceleration mechanisms (Hudson et al. 2008), and electron loss processes (Millan and Thorne 2007). A concise discussion of the importance of wave-particle interactions was recently provided by Thorne (2010). The present review will focus on observations of electrons and protons in the $\mathrm{MeV}$ energy range and how these observations relate to current models of acceleration. We do not attempt to address energization of the lower energy $(\leq 100$ 's $\mathrm{keV})$ ring current population or acceleration occurring in the plasma sheet. We first provide a historical overview in order to put into context our current understanding of the radiation belts and address the origin of radiation belt particles (Sect. 1). In Sect. 2, the physics of particle trapping is briefly reviewed and particle lifetimes discussed. Section 3 gives a detailed overview of our modern view of the radiation belts based on fifty years of collected observations. In Sect. 4, we give a brief review of acceleration mechanisms, and in Sect. 5, provide a summary and briefly discuss applications to other planetary systems.

\subsection{Discovery of Earth's Radiation Belts}

In the late hours of January 31, 1958, the first U.S. Satellite, Explorer I, was launched into an elliptical orbit with $2500 \mathrm{~km}$ apogee. The spacecraft carried three science experiments including a Geiger-Müller counter designed to measure the cosmic ray intensity as a function of magnetic latitude. Data were periodically downlinked when the spacecraft was over 
Fig. 11959 sketch of the intensity structure of the radiation belts based on Pioneer III measurements. The numbers represent contours of constant counting rate from the Geiger-Müller counter.

Reprinted by permission from MacMillan Publishers Ltd: Nature (Van Allen and Frank 1959a), copyright 1959

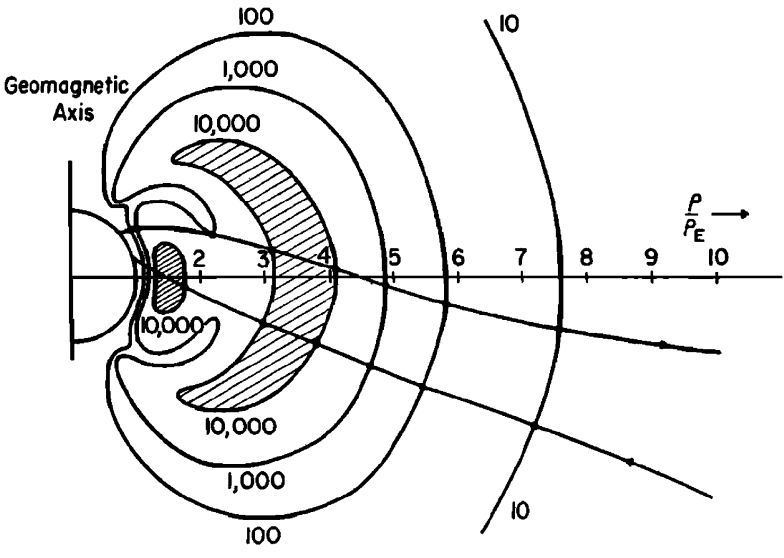

an available ground station and it was observed that the Geiger counter worked sometimes, but the count rate dropped to zero at other times. The experimenters suspected that the instrument was saturating due to excessively high count rates, and this was soon confirmed when Explorer III was successfully launched on March 26, 1958 (Explorer II had failed on launch). Explorer III carried the same Geiger-Müller counter plus a tape recorder, revealing what had happened on Explorer I. At low altitude, cosmic rays were measured at the expected count rate of $\sim 30$ counts/second. As the satellite rose, the count rate began to increase, but then fell abruptly to zero as the instrument saturated due to an extremely high rate of incident radiation (Stern 1996). This marked the discovery of Earth's radiation belts.

Slightly ahead of the U.S. efforts to launch artificial satellites, the Soviet Union launched Sputnik 1 on October 4, 1957, and less than a month later, Sputnik 2 was launched on November 3, 1957. Sputnik 2 carried a radiation detector similar to that of the Explorer satellites. The increased radiation levels of the radiation belts were detected by the instrument before Explorer I was even launched, but only when the satellite was near apogee at high enough altitude and over Australia. Because the Soviets could not directly downlink the high altitude data, the data were not immediately analyzed. After Van Allen reported the discovery from Explorer III, the Sputnik data were examined and the presence of the radiation belts confirmed by Vernov (Dessler 1984).

Geiger-Müller tubes were subsequently carried on Sputnik 3 (Vernov et al. 1959b), Explorer IV (Van Allen et al. 1959a), and Pioneer III (Van Allen and Frank 1959a) which measured the radiation to a radial distance of $107,400 \mathrm{~km}$, establishing the existence of two distinct zones separated by a region devoid of particles (Fig. 1). A comprehensive bibliography of the earliest papers through May 1959 is given in Van Allen (1959a). Van Allen summarized his early observations in Van Allen (1959c), and also wrote a popular account in Scientific American (Van Allen 1959b).

Within a few years after the discovery, a picture of the radiation belts had emerged which is surprisingly similar to our current understanding. The basic structure and composition of the belts was relatively well understood, including the fact that the inner zone is relatively stable in time while the outer zone varies in relation to solar activity. The first hint of this variability was based on Pioneer IV measurements obtained in March 1959 following a period of intense auroral activity. The outer zone intensity was significantly higher than that observed during geomagnetically quiet times by Pioneer III in December 1958 and the Soviet Cosmic rocket in January 1959. The inner zone was observed to be relatively stable during this time (Van Allen and Frank 1959b). A more thorough analysis of Explorer IV 
data showed that both the average energy and intensity in the outer zone increased following magnetic storms. The increase was often preceded by a large decrease in the intensity (Rothwell and McIlwain 1960). A nice review describing the state of the field in 1965 is given in Hess et al. (1965). Their Table 1 (Hess et al. 1965) lists parameters of both American and Russian experiments conducted between 1957-1964.

\subsection{Origin of Radiation Belt Particles}

Data from Explorers I and III revealed that the intensity of radiation rises abruptly above $1000 \mathrm{~km}$ altitude in a way that is inconsistent with cosmic rays. Although the identity of the radiation was initially uncertain, Van Allen interpreted this altitude profile as evidence that the radiation was affected by Earth's magnetic field and therefore must consist of charged particles (Van Allen et al. 1959a). Although natural radiation belts weren't expected at the time, the theory of particle motion and confinement in a magnetic field was well understood based on early work by Alfvén (1939) and Størmer (1955) among others (e.g., Spitzer 1952; Rosenbluth and Longmire 1957). In 1957, prior to the discovery of the natural radiation belts, Christofilos had proposed an experiment to detonate several small atomic bombs in the upper atmosphere in order to study the trapping of the beta decay electrons from fission fragments (Christofilos 1959a, 1959b). The Argus experiment was carried out in 1958 and is discussed in more detail in Sect. 2 below.

Early observations suggested that the nature of radiation in the inner and outer zones is different both in terms of the composition and temporal variability (Van Allen 1959c). As mentioned previously, the outer zone varies with solar activity while the inner zone is relatively stable, and this was interpreted as evidence for different sources for the two populations (Van Allen and Frank 1959b; Rothwell and McIlwain 1960). When considering the energetic particle population, the two-zone structure of the radiation belts (Fig. 1) holds for electrons, but protons are distributed radially in a more continuous fashion. Additionally, the energy properties of both electrons and protons differ between the inner and outer zones.

\subsubsection{Inner Zone}

Protons with energies extending above several hundred $\mathrm{MeV}$ are concentrated within about one Earth radius from the surface of Earth. In this region, the bulk of electrons has energies below $1 \mathrm{MeV}$, but electrons can be found with energy as high as $5 \mathrm{MeV}$. The lack of variability in the inner zone indicates a steady source and long particle lifetimes which lead to a relatively stable equilibrium distribution of particles.

Cosmic Ray Albedo Neutron Decay (CRAND) was first proposed independently by Singer (1958), Vernov et al. (1959a), and Kellogg (1959) as a possible source for the trapped radiation, and further developed by Hess (1959) and Freden and White (1960), among others. The average incident galactic cosmic ray flux reaching Earth is roughly 2 particles $/ \mathrm{cm}^{2} \mathrm{~s}$ (e.g. the International Organization for Standardization ISO-15390 GCR model), which could potentially contribute to the trapped population in several ways. Quasi-trapping of the cosmic ray protons, direct production of charged particles by cosmic ray interaction in the atmosphere, and decay of $\pi$ mesons produced in the atmosphere were all shown to be insufficient sources (e.g., Hess 1962). However, cosmic ray protons also produce neutrons when they collide with oxygen and nitrogen in the atmosphere. Selesnick et al. (2007) have calculated the vertical neutron flux as a function of energy using a Monte Carlo approach and find general agreement with observations from Kanbach et al. (1974) and Preszler et al. (1976) made over a limited energy range (see Fig. 6 of Selesnick et al. 2007). 
When a neutron escapes the atmosphere, it is free to move on a ballistic trajectory unaffected by the magnetic field. However, the neutron will decay into an electron, a proton, and an anti-neutrino with a mean lifetime of just under 15 minutes. The proton has kinetic energy approximately equal to the neutron energy. The electrons produced will have a typical beta-decay spectrum. Both electrons and protons can be trapped in the magnetic field. For protons, the trapping efficiency was estimated to be $\sim 30 \%$ since some protons will have small pitch angles and be immediately lost to the atmosphere (Lenchek and Singer 1962).

Comparison of observations with CRAND theory shows that CRAND alone has serious deficiencies, predicting too few protons with low magnetic moments (i.e. energies less than $\sim 50 \mathrm{MeV}$ ) (e.g., Farley et al. 1970). Further work by Farley and Walt (1971) combined the CRAND source with atmospheric losses and radial diffusion from an outer boundary at $L=1.7$ (a radial distance of 1.7 Earth radii from the center of Earth). They adjusted the diffusion coefficient to obtain the best match with observations and found that this model could reproduce the observed profiles. More recently, Selesnick et al. (2007) have produced a comprehensive model of the inner radiation belt protons. The proton intensity below $100 \mathrm{MeV}$ was found to be dominated by solar protons for $L>1.3$, while the CRAND source is sufficient at higher energies. Above $100 \mathrm{MeV}$, particle lifetimes can exceed 1000 years, thus the long-term variation of Earth's magnetic dipole moment was also investigated.

The first solid evidence for heavy ions trapped in Earth's magnetic field was obtained in the early 1990's (Grigorov et al. 1991). Heavy ions are observed near $L=2$ with energies greater than $10 \mathrm{MeV} /$ nucleon, and the source is anomalous cosmic rays-interstellar particles that have been energized in the heliosphere (e.g., Mewaldt et al. 1996).

The energy spectrum expected for CRAND electrons is a beta decay spectrum weighted by the energy-dependent particle lifetime which is determined by Coulomb collisions in the atmosphere. The maximum energy of a beta decay electron is $780 \mathrm{keV}$, however, electrons with energies above $1 \mathrm{MeV}$ have been observed in the inner zone (Hess 1962). Moreover, at lower energies, the spectrum is not consistent with CRAND (Singer 1962). CRAND may still provide a source for electrons but some acceleration mechanism must be acting to energize them. Alternatively, electrons may be moved into the inner zone from larger radial distances.

\subsubsection{Outer Zone}

Moving radially outward from Earth, the intensity of protons in the outer zone is much higher than in the inner zone yet the neutron albedo is weaker, thus the CRAND mechanism is not sufficient to explain their origin (e.g., Hess et al. 1965). Average proton energies are also much lower in the outer zone because the gyro-radius of protons becomes quite large as the magnetic field strength decreases with distance from Earth. For example, even at $2 R_{E}$, the maximum trappable energy of protons is $\sim 75 \mathrm{MeV}$ (Lenchek and Singer 1962) and it decreases at larger distances as the field becomes weaker.

However, the outer zone is dominated by electrons (e.g., Cladis et al. 1961) and distinguished by a high degree of variability (Fig. 2). It has long been known that the magnetic field of Earth becomes severely disturbed due to solar activity (e.g., Crawford 1959), with periodic geomagnetic activity recurring roughly once per month due to high speed solar wind streams that emanate from coronal holes, and episodic activity due to coronal mass ejections (CMEs). Thus, the persistence of the outer zone requires an unabating source. Immediately after the radiation belts were discovered, Gold (1959) considered direct injection of particles by magnetic clouds from the Sun as a potential source of electrons. Kellogg (1959) further investigated direct injection of solar particles, with subsequent radial diffusion leading to their adiabatic energization. A comprehensive early review of energization 


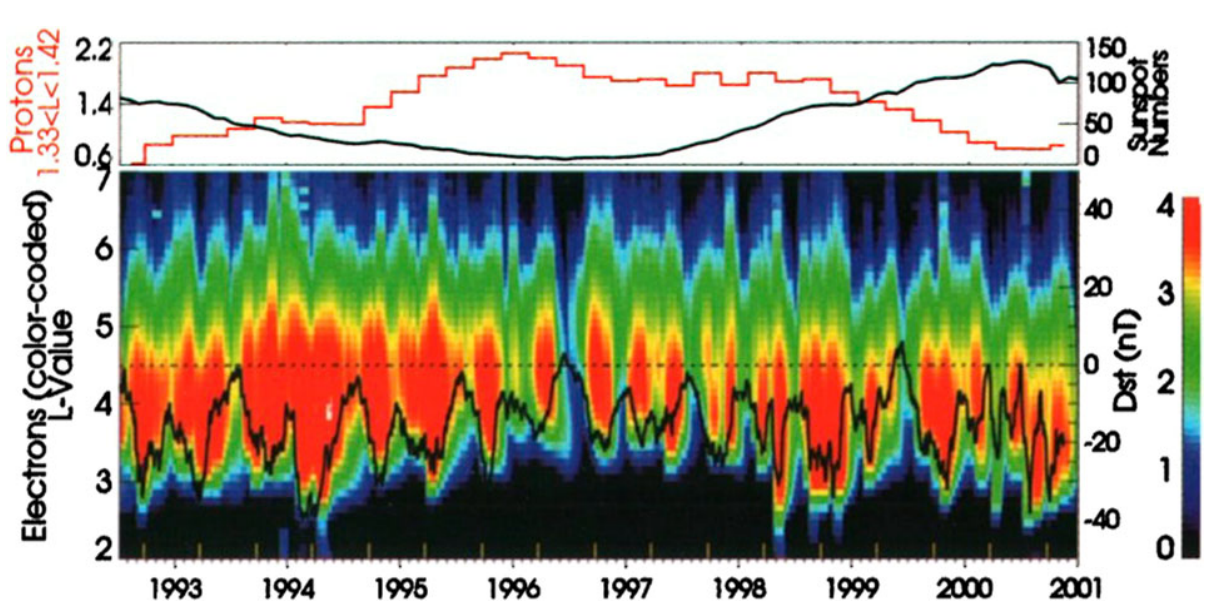

Fig. 2 SAMPEX 19-27.4 MeV inner zone proton intensity (top) and outer zone 2-6 MeV electron intensity (bottom), along with window-averaged sunspot number (top) and Dst index (bottom), shown over solar cycle 23. Reproduced from Li et al. (2001), copyright 2001, American Geophysical Union

by radial diffusion was provided by Tverskoy (1969). Acceleration of an existing particle population, for example by Fermi acceleration, was also considered early on (e.g., Crawford 1959).

Before discussing acceleration mechanisms in detail, it is first essential to establish whether the particles are accelerated internally from very low energies or whether they are directly injected from an external source. Electrons with energies below $\sim 40 \mathrm{MeV}$ are observed in interplanetary space near Earth and were originally thought to be secondaries produced by higher energy cosmic rays. However, measurements by Pioneer 10 showed that the spectral shape of electrons $(0.2-8 \mathrm{MeV})$ within $1 \mathrm{AU}$ of Jupiter was remarkably similar to that inside Jupiter's magnetosphere. Measurements of 3-12 MeV electrons with IMP-3,4, and 5 near Earth showed a 13-month periodicity expected for a favorable magnetic connection between Earth and Jupiter (Teegarden et al. 1974). The same periodicity was observed between 0.2-2.5 MeV (Mewaldt et al. 1976) and 1-6 MeV (Krimigis et al. 1975 ) with IMP-7 and IMP-8. Thus the relativistic electron population below $\sim 25 \mathrm{MeV}$ in interplanetary space near Earth is comprised of Jovian electrons, as well as solar electrons, and magnetospheric electrons (below $1 \mathrm{MeV}$ ) that were accelerated in the magnetotail.

These interplanetary electrons, then, are an obvious potential source of particles for the radiation belts. This was first suggested by Baker et al. (1979a) who examined geosynchronous electron data between $200 \mathrm{keV}-2 \mathrm{MeV}$ over a period of two years and found some evidence of a 13-month periodicity in the trapped flux. They suggested that the Jovian electrons could gain access to the magnetosphere and become part of the plasma sheet population where they could subsequently be injected into the radiation belts. A later study compared the 6-10 MeV interplanetary electron flux with the $0.2-7 \mathrm{MeV}$ flux at geosynchronous orbit over a longer time interval of eight years and found no clear correlation (Christon et al. 1989). This study concluded that, although the interplanetary electrons could be a reservoir for particles that contribute to the trapped population, the rapid enhancements observed at geosynchronous orbit are not due to increases in the interplanetary electron flux, and the enhancements must therefore be due to internal processes. Baker et al. (1996) provide a review of external sources and conclude that the bulk of relativistic electrons is accelerated within the magnetosphere. 
Acceleration of a lower energy source population is thus required to explain the rapid variability of radiation belt electrons, but what is that lower energy source? Li et al. (1997) considered the solar wind high energy tail or "super-halo" (Lin 1998), and showed that it did not have sufficient phase space density to account for the radiation belt electrons, assuming adiabatic acceleration by radial diffusion. It is now widely believed that the source of electrons is in Earth's magnetotail. Particles from the $\sim \mathrm{keV}$ plasma sheet population are energized and injected into the inner magnetosphere during substorm dipolarization events (e.g., Baker et al. 1981; Apatenkov et al. 2007; Dubyagin et al. 2011; Runov et al. 2011). Substorm injections extending above a few hundred $\mathrm{keV}$ are rare and the appearance of high energy electrons at geosynchronous orbit is typically delayed by 2-3 days following the main phase of a goeomagnetic storm (Baker et al. 1986). Thus, additional acceleration is required to explain the existence of energetic electrons which can extend to energies exceeding $10 \mathrm{MeV}$.

In summary, protons in the inner zone (below $L \sim 1.3$ ) are consistent with the CRAND source, while solar protons are an important source at larger radial distances at energies below $100 \mathrm{MeV}$. CRAND likely contributes to the electron population in the inner zone, but is not sufficient to explain either the intensity of electrons or the existence of electrons above $800 \mathrm{keV}$. In the outer zone, the rapid variability of relativistic electrons and the lack of an established external source implies an efficient acceleration mechanism acts on magnetospheric electrons, accelerating them to multiple $\mathrm{MeV}$ energies on a timescale as short as a few hours. Possible acceleration mechanisms are discussed in more detail in Sect. 4 below.

\section{Particle Trapping and Lifetimes}

In order to understand the context in which particles are accelerated to high energies in the radiation belts, we must first understand the basic physics of trapping and the processes that govern the lifetime of particles in this region. Particle lifetimes place important constraints on acceleration mechanisms that produce the trapped radiation. In principle, even a weak or inefficient acceleration mechanism could explain the presence of high energy particles in the magnetosphere if the particle lifetimes are long enough. In addition, the non-dipolar and dynamic nature of the magnetic field places constraints on the region in which particles can be stably trapped. Improvements in available computational tools have allowed for recent advances in our understanding of particle dynamics in a realistic magnetic field. This section provides a brief review of these topics.

\subsection{Trapped Particle Motion and Adiabatic Invariants}

The confinement of particles in a dipole magnetic field was first discussed by Størmer and further explored by Alfvén, who looked in detail at particle trajectories (e.g., Elliot 1963). The dynamics of charged particles in a quasi-dipole magnetic field can be formulated in terms of adiabatic theory, first applied by Northrop and Teller (1960). Here we provide a brief overview, but the reader is referred to e.g., Schulz and Lanzerotti (1974) for a detailed discussion.

In a dipole magnetic field, charged particles undergo three types of periodic motion: gyration, bounce along the field line between mirror points, and azimuthal gradient-curvature drift. For each of these motions, the trajectory of a particle in phase space is closed, and the action variable is defined by the area of the orbit in phase space

$$
J_{i}=\oint P_{i} d q_{i}
$$



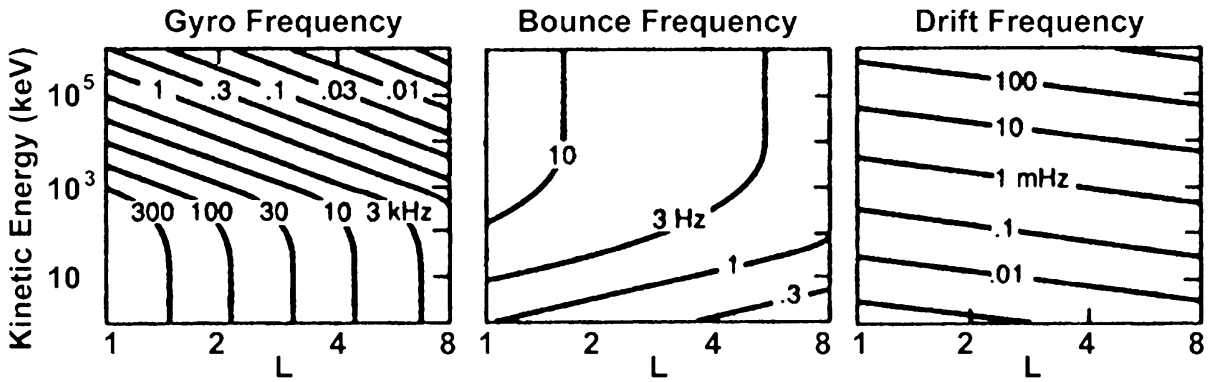

Fig. 3 Frequencies of gyration, bounce, and drift motion as a function of energy for equatorially mirroring electrons in a dipole field at Earth. Adapted from Schulz and Lanzerotti (1974)

where $P_{i}$ is the canonical momentum, and $q_{i}$ is the generalized coordinate. For a slowly varying system (relative to the frequency of motion), the action is an adiabatic invariant, approximately conserved.

In the radiation belts where the field is quasi-dipolar, the three periodic motions have timescales that are typically separated by orders of magnitude (Fig. 3), and are thus decoupled. The first action variable is given by

$$
J_{1}=\frac{c}{e} \frac{\pi p_{\perp}^{2}}{B}
$$

where $e$ is the charge, $c$ is the speed of light, $p_{\perp}$ is the momentum perpendicular to the local magnetic field, and $B$ is the magnetic field strength. Making use of the constancy of $c, e$, and the particle rest mass, $m$, the related quantity, $\mu$, is defined to be the "first adiabatic invariant"

$$
\mu=\frac{p_{\perp}^{2}}{2 m B}
$$

which is related to the magnetic moment of the particle. In a static magnetic field, the particle energy is also conserved which, together with constant $\mu$, implies that the particle motion satisfies

$$
\frac{\sin ^{2} \alpha}{B}=\text { constant }
$$

Here we have defined the pitch angle, $\alpha$, to be the angle between the velocity vector of the particle and the local magnetic field direction. Note that the pitch angle increases as the particle moves along the magnetic field line away from the equator, reaching $90^{\circ}$ at the mirror point. Throughout the remainder of this paper, "equatorial pitch angle" will refer to the pitch angle of the particle as it passes through the equatorial plane.

The second adiabatic invariant is given by

$$
J=\oint p_{\|} d s
$$

where $p_{\|}$is the momentum parallel to the magnetic field, $s$ is the coordinate along the magnetic field line, and the integration is carried out along the bounce trajectory. Conceptually, the second adiabatic invariant is related to the length of the field line between mirror points. More precisely, one can show that, together with constant $\mu$ and $p$, the length

$$
\frac{J}{p}=2 \int_{s_{m}}^{s_{m}^{\prime}} d s \sqrt{1-\frac{B(s)}{B_{m}}}
$$


is constant as the particle drifts in longitude, where $B_{m}$ is the magnetic field strength at the mirror point, and $s_{m}$ and $s_{m^{\prime}}$ are the coordinates of the two mirror points.

Finally, the third adiabatic invariant is

$$
J_{3}=\frac{e}{c} \Phi
$$

where $\Phi$ is the magnetic flux enclosed by the drift shell

$$
\Phi=\frac{-2 \pi B_{0} R_{E}^{2}}{L}
$$

Here $L$ is the distance from the dipole center to the location where the field line on which the particle bounces crosses the equator, measured in units of Earth radii, $R_{E} . B_{0}$ is the magnetic field strength at the surface of Earth. In the real magnetic field which deviates from a perfect dipole, it is useful to recast the third invariant in terms of a generalized $L$-value (Roederer 1970)

$$
L^{*}=\frac{2 \pi B_{0} R_{E}^{2}}{|\Phi|}
$$

where the magnetic flux through the drift orbit can be numerically calculated, assuming the drift path is known. $L^{*}$ is physically interpreted as the radial distance at which a particle would cross the equator if all non-dipole components of the magnetic field were adiabatically turned off.

The three adiabatic invariants describe the unperturbed motion of a particle in a quasidipolar magnetic field. The observed variability of the trapped population suggests that one or more of the adiabatic invariants is frequently violated in the radiation belts. Nevertheless, it is useful to organize data in terms of the three invariants in order to study deviations from adiabatic motion. In particular, particle acceleration implies violation of one or more invariants, which is further discussed in Sect. 4.

\subsection{Particle Dynamics in a Realistic Magnetic Field}

The magnetic field in the radiation belt region is often approximated by a dipole field or a model such as the International Geomagnetic Reference Field which includes higher order multipole terms but does not include external current systems. Under typical conditions, this approximation is quite good near the surface of Earth where the field is strong, for example within a few Earth radii. However, the field can deviate from a dipole quite substantially; at geosynchronous orbit, the effects are important even for quiet-time conditions. The nondipolar effects become substantial well inside of GEO during periods of strong geomagnetic activity. An increase in solar wind dynamic pressure causes a significant compression on the dayside, while momentum transferred from the solar wind stretches the nightside field into a tail-like structure. A buildup of hot plasma pressure in the inner magnetosphere, called the ring current, contributes significantly to the field during magnetic storms, causing a decrease in the field strength as measured by ground-based magnetometers. Finally, the magnetic field can be very dynamic, responding to changes in the solar wind driver. The non-dipolar and dynamic nature of the magnetic field in the radiation belts has important implications for the particle motion.

Drift shell splitting occurs when stably trapped particles with different pitch angles follow different drift trajectories due to the asymmetry in the magnetic field. Figure 4 shows the full trajectories for an equatorially mirroring $\left(90^{\circ}\right.$ pitch angle) particle (red) and a 


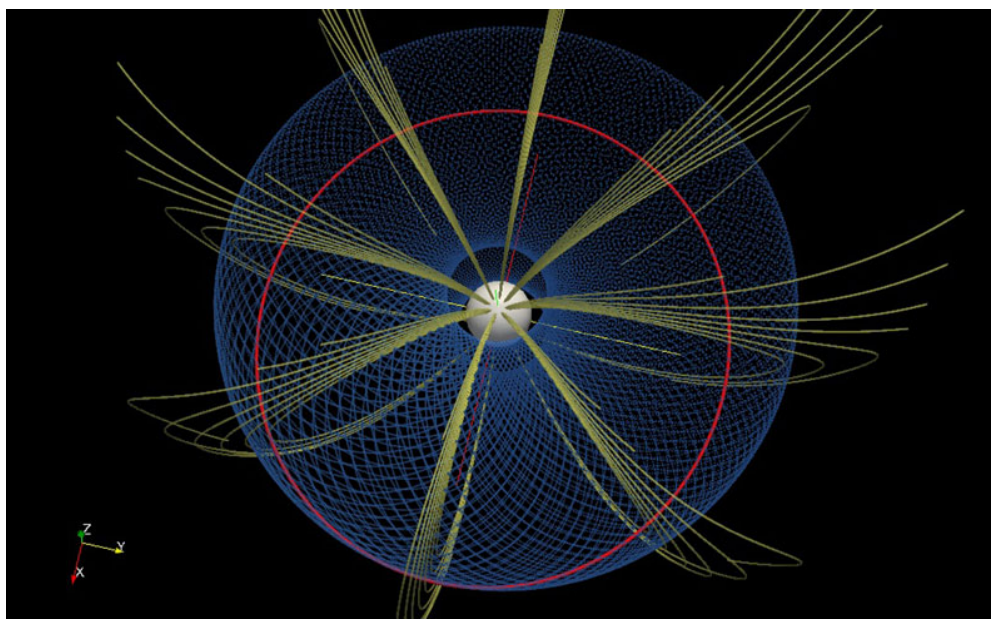

Fig. 4 Drift shell splitting is illustrated in this test particle simulation. Looking almost directly down at the equatorial plane, trajectories for a $90^{\circ}$ (red) and small equatorial pitch angle (blue) particle are shown. Noon is towards the bottom of the figure along the positive $x$-axis. Figure courtesy A.Y. Ukhorskiy

particle with small equatorial pitch angle (blue). To conserve the first and second adiabatic invariants, the equatorial particle must follow a contour of constant magnetic field strength: $J=0=$ constant implies $\alpha=90^{\circ}=$ constant (no parallel momentum), thus we see from Eq. (4) that $B$ must also be constant on the particle trajectory. Because the magnetic field is stronger on the dayside where it is compressed, the particle moves outward. Particles with smaller equatorial pitch angle, on the other hand, mirror close to the Earth where the field is stronger and less distorted by the solar wind. The mirror points (where $p_{\perp}^{2}=p^{2}$ ) for this particle also lie on a contour of constant $B$ in order to conserve the first adiabatic invariant. This trajectory (Fig. 4) is more symmetric and nearly circular, due to the fact that the field is less distorted near Earth. This is a conceptual picture to explain the origin of the drift-shell splitting effect, but in general particles will move on trajectories that conserve $\mu$ and $J$, and these trajectories turn out to be dependent on $\mu$ and $J$ for a non-symmetric field. This effect has important implications for spacecraft measurements of pitch angle distributions. The two particles shown in Fig. 4 pass through the same equatorial location near noon. In this example, a spacecraft measuring the pitch angle distribution would measure both particles at noon, but would not measure the same two particles on the nightside where their drift shells have separated substantially. This is one explanation for observed butterfly pitch angle distributions (e.g., Roederer 1967; Schulz and Lanzerotti 1974).

Another effect of the dayside compression of the field was first recognized by Northrop and Teller (1960), and later discussed by Roederer, Shabansky, and others (e.g., Roederer 1970; Shabansky 1971). In a dipole field, particles exhibit bounce motion which is symmetric around the equator where there is a local minimum in the magnetic field strength. In the compressed field at large enough equatorial distance, there are two local minima that occur at higher latitude, off of the equator. Particles drifting into the dayside will start to bounce around one of these minima rather than bouncing symmetrically around the equator. This occurs because they reach their mirror field strength and are reflected before reaching the equator. Since there are two of these minima, the drift orbit bifurcates such that a particle may bounce about the minimum in the northern hemisphere or 
the minimum in the southern hemisphere, depending on its bounce phase when it first encounters this compressed region. The so-called "drift orbit bifurcation" is pitch-angle dependent, affecting particles with larger pitch angles more strongly. Although this effect has been known for a long time, the extent to which the field is distorted was not appreciated. Development of empirical models such as the Tsyganenko magnetic field models (e.g., Tsyganenko and Sitnov 2005), based on decades of observations, has allowed for recent test particle simulations to further explore these effects (Öztürk and Wolf 2007; Ukhorskiy et al. 2011a). The effects are important; the periods of the bounce and drift motions are no longer well-separated, thus the second adiabatic invariant is not conserved. Moreover, since the particle no longer moves on a closed drift path, the third invariant is no longer well-defined. This effect can be important inside geosynchronous orbit for a range of particle pitch angles, even for moderately active conditions.

Finally, the dynamic nature of the magnetic field has significant effects on particle motion. Variations that occur on the timescale of the particle periodic motions (i.e. gyration, bounce, drift) violate one or more adiabatic invariants. These variations can be plasma waves at a range of frequencies, extending from $\mathrm{mHz}$ (drift timescale) to $\mathrm{kHz}$ (gyration timescale) and beyond. Such variations can lead to energization of particles as is further discussed in Sect. 4, or pitch angle scattering, briefly discussed in the next section. Much slower variations in the magnetic field cause particles to move adiabatically. For example, when the hot plasma pressure builds up in the inner magnetosphere during a storm, the magnetic field strength is reduced, and particles move outward to conserve the magnetic flux through their drift orbit (third invariant). Called the "Dst effect", this can lead to perceived changes in particle intensity as measured by a spacecraft at fixed location and energy, which are reversed when the field returns to its pre-storm value. The Dst effect can also lead to real changes in the trapped particle intensity. If particles move outward far enough, they may find themselves on open drift paths and be lost to the magnetopause (e.g., Ukhorskiy et al. 2006b). The effects of the dynamic field on particles in the bifurcation region are not yet known and are only now being explored with fully three dimensional test particle simulations in realistic fields based on empirical models or MHD simulations.

\subsection{Loss Mechanisms and Particle Lifetimes}

Loss processes help define the structure of the radiation belts. Losses are responsible for the characteristic slot region between the inner and outer electron zones, and the lack of the highest energy protons in the outer zone where the gyro-radius is comparable to the field line curvature. Loss timescales are location- and energy-dependent and generally difficult to measure in-situ since the measured particle flux is the combined product of acceleration, transport, and loss. Particle lifetimes are controlled by several different mechanisms, including Coulomb scattering by the atmosphere at low altitudes, pitch angle scattering into the atmospheric loss cone by plasma waves, curvature scattering where the gyro-radius is comparable to the field-line curvature, and loss through the magnetopause boundary.

Coulomb scattering is important for particles mirroring at low altitudes (below a few hundred km) where they can interact with Earth's atmosphere. Coulomb collisions can lead to pitch angle scattering and energy loss. Modification of the pitch angle distribution has been calculated by solving the Fokker-Planck equation (e.g., MacDonald and Walt 1962; Walt and MacDonald 1962) and using a Monte Carlo approach (e.g., Selesnick 2004).

For particles mirroring at higher altitudes where Coulomb collisions are not important, pitch angle diffusion into the loss cone is dominated by wave-particle interactions. Recent reviews of pitch angle scattering by plasma waves were given by (e.g., Millan 
and Thorne 2007; Shprits et al. 2008b; Thorne 2010). Shklyar and Matsumoto (2009) discuss interactions between charged particles and oblique whistler-mode waves. The slot region between the inner and outer electron zones is due to scattering by whistler mode waves that are generated by a range of mechanisms, including magnetospheric processes, lightning and VLF transmitters (Lyons et al. 1972; Lyons and Thorne 1973; Abel and Thorne 1998). Other waves that may be important for pitch angle scattering include electromagnetic ion cyclotron waves and plasmaspheric hiss. The dynamic and non-dipolar nature of the real magnetic field may significantly reduce the lifetime of particles in the outer parts of the radiation belts, since particles may also be lost to the magnetopause.

For the most part, loss rates have not been sufficiently well quantified with experiments except perhaps in the inner zone and slot region. A unique opportunity to directly measure the particle lifetimes was presented in the 1960's when high altitude nuclear explosions were carried out by both the United States and the Soviet Union. The first of these was the Argus experiment, specifically designed to study the trapping of relativistic electrons. Three small atomic bombs were detonated over the south Atlantic, injecting a host of fission fragment particles into the slot region at an altitude of about $1 R_{E}$. The hypothesis was that electrons with 1-2 MeV energy would survive several hours or more, losing energy only due to Coulomb collisions with the atmosphere. The lifetime was measured by satellite and rocket experiments. The new belt of trapped electrons was found to be remarkably stable spatially, remaining at the same location and not spreading radially. However, the lifetime was found to be shorter than expected from Coulomb scattering alone, and this prompted the suggestion that magnetic variations were contributing to the loss. A rapid drop in intensity was also observed in association with a magnetic storm (Van Allen et al. 1959b). More recently, decay rates were measured after the injection of a new radiation belt in the slot region between $L=1-2$ during the 2003 Halloween Storm period. The loss rate for electrons between 2-6 MeV was found to be highly L-dependent, ranging from 180 days at $L=1.5$ to $\sim 8-35$ days at $L=2$ (Baker et al. 2007). Prior to the formation of the new radiation belt in the slot region, the radiation belts were first rapidly depleted across all $L$-shells during this storm. The radiation belts were then re-populated to very high intensity, but deep inside the slot region (Baker et al. 2004). Thus, extreme events can completely wipe out the radiation belts, allowing us to observe the acceleration timescale over which the belts are re-formed.

In the outer zone, losses during less extreme events can also be quite rapid. Estimates have been made based on observations, for example, Selesnick (2006) analyzed data from the SAMPEX satellite and found that atmospheric losses during the main phase of a storm can deplete the radiation belts in about 1 hour. Other estimates range from hours to days (e.g., Lorentzen et al. 2001; Millan et al. 2002). On the other hand, Turner et al. (2012) recently concluded that loss through the magnetopause was responsible for large decreases in the trapped population during the main phase of storms. The relative role of atmospheric loss versus magnetopause loss is currently not known.

\section{Characteristics of Earth's Radiation Belts}

In this section we summarize the observed characteristics of the trapped radiation including the radial profile, energy spectra, pitch angle distributions, and temporal variability. Any viable models of acceleration, transport, and loss must be able to explain these observed features. 


\subsection{Spatial Distribution}

A number of empirical models of the radiation belts have been developed based on spacecraft data collected over many years. These are primarily used for operational/engineering purposes to define radiation levels, but can also provide an average picture of the general characteristics of the radiation belts. An overview of the trapped electron and proton distributions is shown in Fig. 5 based on the AE-8 and AP-8 solar minimum models (from Elkington et al. 2004). AE-8 and AP-8 are empirical models of electrons and protons in the radiation belts, providing electron and proton fluxes over a wide range of energies and radial distances, and include versions for solar minimum and solar maximum (Vette 1991). Model outputs can be obtained at the Space Physics Data Facility (http://modelweb.gsfc.nasa.gov/models/trap.html). These models were completed in 1983 and are based on earlier models (e.g. AE-5 in the inner zone and AE-4 in the outer zone) and data from multiple spacecraft, including GRS-A, OV1-19, OV3-3, and ATS 5 and 6. An effort is currently underway to develop the next generation models AE9/AP9 based on more recent data from a wide range of spacecraft including CRRES, Polar, LANL GEO, SCATHA, and others. Later versions will also include data from the RBSP and DSX missions.

In Fig. 5, the two zone structure of electrons is apparent with the slot region occurring near $L=2.5$ and becoming more prominent at higher energies. Protons do not show evidence of a slot, rather they are narrowly peaked around $L=1.5$ at the highest energies, and more broadly distributed at lower energies, with the peak shifting to higher $L$ as the energy decreases. Protons with energy above $100 \mathrm{MeV}$ are confined to $L \leq 3$, while protons with energy less than $\sim 1 \mathrm{MeV}$ can be found out to distances of $\sim 7 R_{E}$. The distribution of particles along the field lines is governed by the pitch angle distribution, discussed in Sect. 3.3 below.

\subsection{Energy Distribution}

\subsubsection{Inner Zone}

The energy spectrum of protons between $80-700 \mathrm{MeV}$ was measured by Freden and White (1962), and found to be in general agreement with the CRAND theory, which predicts a power law spectral shape. The proton flux in protons $/ \mathrm{cm}^{2} / \mathrm{s} / \mathrm{MeV}$ is given by (Hess 1962)

$$
\phi=110 E^{-0.72} \text { for } 10<E<80 \mathrm{MeV}
$$

and

$$
\phi=4.2 \times 10^{6} E^{-2.54} \text { for } 80<E<700 \mathrm{MeV}
$$

where the energy, $E$, is measured in $\mathrm{MeV}$. Electron energies extend to $\sim 5 \mathrm{MeV}$ in the inner zone, significantly higher than expected from CRAND.

\subsubsection{Outer Zone}

Electrons are oftentimes observed with energies above $10 \mathrm{MeV}$ in the outer zone (e.g., Baker et al. 1986), and electrons with energies as high as $50 \mathrm{MeV}$ have been reported in association with very strong CME-driven geomagnetic storms (Blake et al. 1992). Below about $300 \mathrm{keV}$, the spectrum has been well fit by an exponential with e-folding energy around $50 \mathrm{keV}$ for both electrons and protons (Baker et al. 1982). These particles are thought to be a seed 

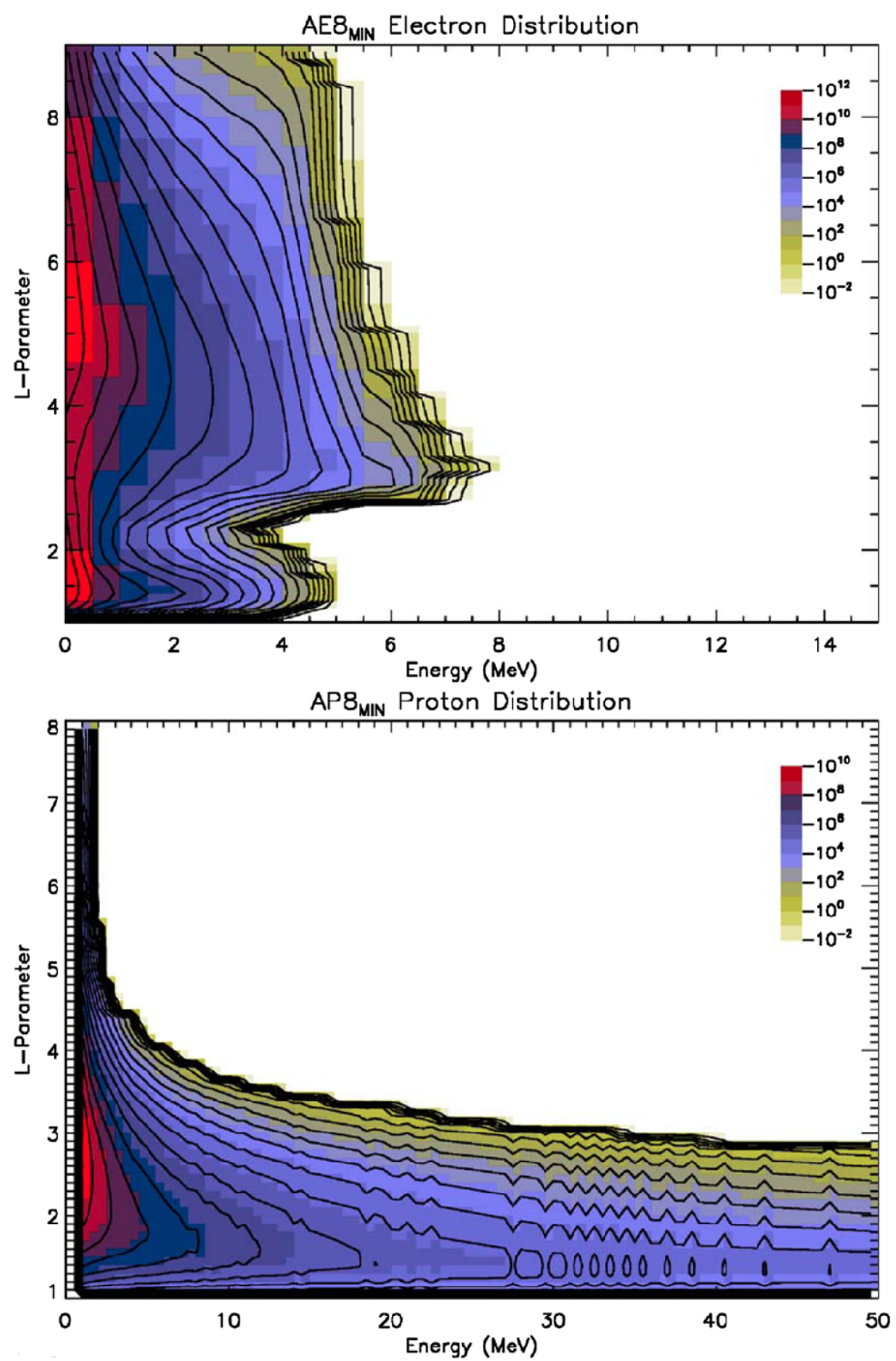

Fig. 5 Integral electron (top) and proton (bottom) fluxes on a relative scale as a function of energy and radial distance ( $L$-parameter) as calculated using the empirical radiation belt models AE- 8 and AP-8. Reprinted from the Journal of Atmospheric and Solar-Terrestrial Physics, Vol. 66, by Elkington et al. (2004), "Physical models of the geospace radiation environment", pp. 1371-1387, copyright (2004), with permission from Elsevier 
population for the higher energy radiation belt particles. Baker et al. (1979b) examined the spectrum of protons up to $\sim 1 \mathrm{MeV}$ during large enhancements of the $>0.3 \mathrm{MeV}$ proton flux and found it was well fit by very steep power law distributions with power law index between $\sim-5$ and -10 .

In general, particle distributions in the magnetospheres of Earth and the outer planets have been successfully represented by a modified kappa distribution which consists of a Maxwellian core with additional power law tails at higher energies (Mauk and Fox 2010). In this representation, the differential intensity (in $/ \mathrm{cm}^{2} / \mathrm{sr} / \mathrm{s} / \mathrm{keV}$ ) is given by:

$$
I(E, \alpha)=\frac{C E\left[k T\left(\gamma_{1}+1\right)+E\right]^{-\left(\gamma_{1}+1\right)}}{1+\left(\frac{E}{E_{0}}\right)^{\gamma_{2}}} \sin ^{2 S}(\alpha)
$$

where $E$ is the energy in $\mathrm{keV}$, the constants $C, E_{0}, k T, \gamma_{1}$, and $\gamma_{2}$ are fitting parameters for the energy distribution, and $S$ is the anisotropy parameter for pitch angle, $\alpha$. The denominator characterizes a break in the spectrum near the energy $E_{o}$ (Mauk et al. 2004).

In the outer zone between $L=4-6$, CRRES data show that $k T \sim 0$ for $0.1-5 \mathrm{MeV}$ electrons, whereby Eq. (10) reduces to (for equatorially mirroring particles)

$$
I\left(E, \alpha=90^{\circ}\right)=\frac{C E^{-\gamma_{1}}}{1+\left(\frac{E}{E_{0}}\right)^{\gamma_{2}}}
$$

with $\gamma_{1} \sim 0.9-1.3, E_{0} \sim 1.3-1.7 \mathrm{MeV}$, and $\gamma_{2} \sim 5.7-7.0$ (e.g. Mauk and Fox 2010, Table 1). In other words, the energy spectrum for outer zone electrons is a power law that goes roughly as $1 / E$ at low energies, becoming significantly steeper at a transition energy between 1-2 $\mathrm{MeV}$. The characteristic $E^{-1}$ spectral shape at low energies was also found by Meredith et al. (2002) during a single geomagnetic storm and in the earlier work of Davidson et al. (1988). The $E^{-1}$ spectrum is consistent with the trapping limit discussed by Kennel and Petschek (1966) (e.g., Mauk and Fox 2010). The spectral break near $\sim 1 \mathrm{MeV}$, where the spectrum becomes much steeper was predicted by Schulz and Davidson (1988). Similar power law spectra with a break near $1 \mathrm{MeV}$ were used by Vampola (1996), but they point out that not all energy spectra are well fit with this model. In an earlier report, Vampola (1971) showed examples of spectra near $L=3.5$ that were not simple power laws, and comment that a more complex energy spectrum was the result of a longer electron lifetime at higher energies.

\subsection{Pitch Angle Distribution}

Electron pitch angle distributions in the inner zone are primarily peaked near $90^{\circ}$. When high energy particles are injected into the slot region following a storm, the pitch angle distributions are typically flat except for a well-defined loss cone. In the outer zone, observed pitch angle distributions are strongly dependent on local time (Fig. 6). This is thought to be largely due to asymmetries in the magnetic field discussed above. In the pre-noon sector, distributions are peaked near $90^{\circ}$, and more strongly peaked for higher energy electrons. In the afternoon and pre-midnight sectors, butterfly distributions that are peaked near $30^{\circ}-$ $40^{\circ}$, are observed. In the early morning sector, a variety of distributions can be observed, ranging from peaked near $90^{\circ}$ to isotropic (West et al. 1973). A flattening of the pitch angle distribution in association with electron energization events in 2002 was reported by Kanekal et al. (2005). The isotropization in these events was attributed to local acceleration by waveparticle interactions. 
Fig. 6 Summary of equatorial pitch angle distributions observed by Ogo 5. Reproduced from West et al. (1973), copyright 1973, American Geophysical Union

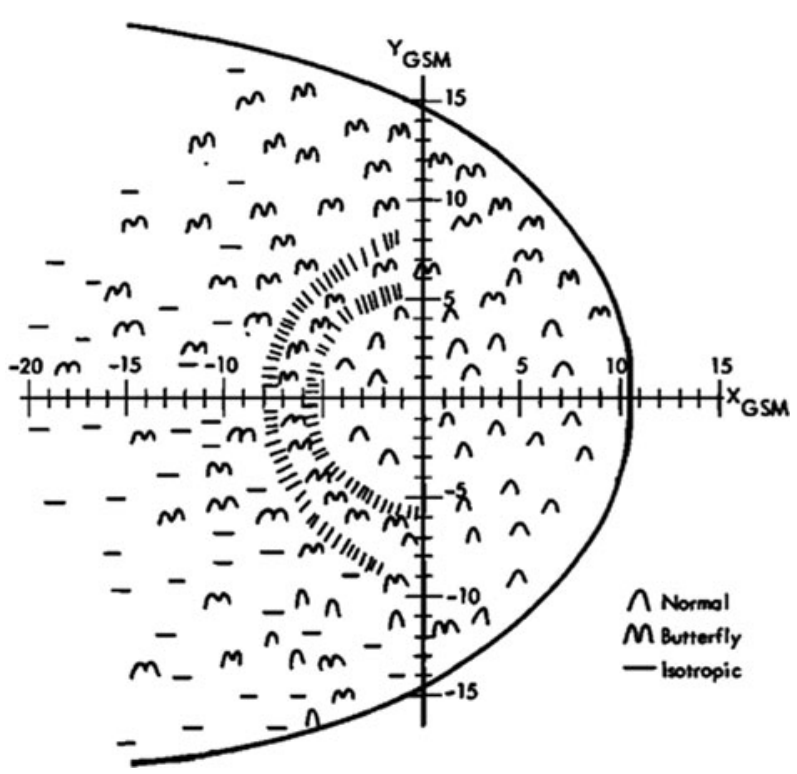

Equatorial pitch angle distributions are often modeled using the function $\sin ^{n} \alpha$. Mauk and Fox (2010) recently found $n$ often increases with energy at geosynchronous orbit, ranging from $\sim 0.5-2.5$. This obviously is not a good model when butterfly distributions exist, but otherwise is a fairly good parameterization. Vampola (1996) reported very similar values for $n$ between 1-2 for the bulk of the outer zone, but as high as 5 between $L=3-4$.

\subsection{Temporal Variability}

As mentioned previously, the outer zone is highly variable, and particle intensities can change by several orders of magnitude on timescales as short as minutes. More typically, the relativistic electron intensity gradually increases in the 2-3 days following a geomagnetic storm (e.g., Baker et al. 1990). Only about $50 \%$ of storms lead to an overall increase in intensity (Reeves et al. 2003). A significant number of studies have tried to determine the conditions that drive these enhancements. Paulikas and Blake (1979) found a strong correlation between solar wind speed and the geosynchronous energetic electron flux, with the electron flux peaking 1-2 days after the arrival of a high speed solar wind stream. The delay was found to be energy dependent, at roughly one day for 140-600 keV particles, and 2 days for $>3.9 \mathrm{MeV}$ particles. More recently, Reeves et al. (2011) conducted a follow-up study, examining geosynchronous data over a twenty year period, spanning nearly two solar cycles. They also found a clear relationship between electron intensity and solar wind speed, but one that is not linear and is dependent on the solar wind speed.

Comparison of simultaneous data from multiple satellites shows that the time variations in the trapped electron intensity are coherent across the outer zone. The temporal variability is also strikingly similar at high and low altitudes, suggesting that the acceleration process leads to an isotropic pitch angle distribution or that some other scattering occurs very quickly after the particles are accelerated (Kanekal et al. 2001). 


\section{Particle Acceleration in the Radiation Belts}

\subsection{Introduction}

In the heart of the radiation belt where the field is a quasi-dipole and characteristic timescales of the particle motion are well separated (Fig. 3), acceleration in the radiation belts can be divided into two categories dubbed "global mechanisms" and "local mechanisms". The former refers to global (radial) transport of electrons, while the latter refers to acceleration occurring at a fixed radial location (e.g. due to wave-particle interactions). Radial transport across magnetic L-shells violates the third adiabatic invariant while conserving the first and second invariants (Sect. 2), as long as it occurs much more slowly than the particle bounce motion. Local acceleration by wave-particle interactions may violate the first and/or second adiabatic invariants, leading to a change in particle energy and/or pitch angle.

The relative importance of the "global" versus "local" mechanisms is not yet firmly established, though it is now believed that the combined effects of "global" and "local" mechanisms working together give rise to the observed enhancements of radiation belt electrons. Williams et al. (1968) first suggested this dual process, where acceleration occurs deep inside the radiation belts by violation of the first or second invariant. The energized electrons then diffuse radially inward and outward to populate other L-shells. Horne (2007) renewed this idea, suggesting that $1-300 \mathrm{keV}$ particles injected from the magnetotail excite plasma waves that can further energize electrons to higher energies. The $\sim \mathrm{MeV}$ electrons can then be diffused both outward and inward to populate the rest of the outer zone (Fig. 7). In this section, we briefly review acceleration by both radial transport and by wave-particle interactions. Recent reviews written by Shprits et al. (2008a, 2008b) address both of these, in particular focusing on modeling of these effects using a quasi-linear diffusion approach. Here we also briefly consider the importance of non-diffusive and non-linear effects.

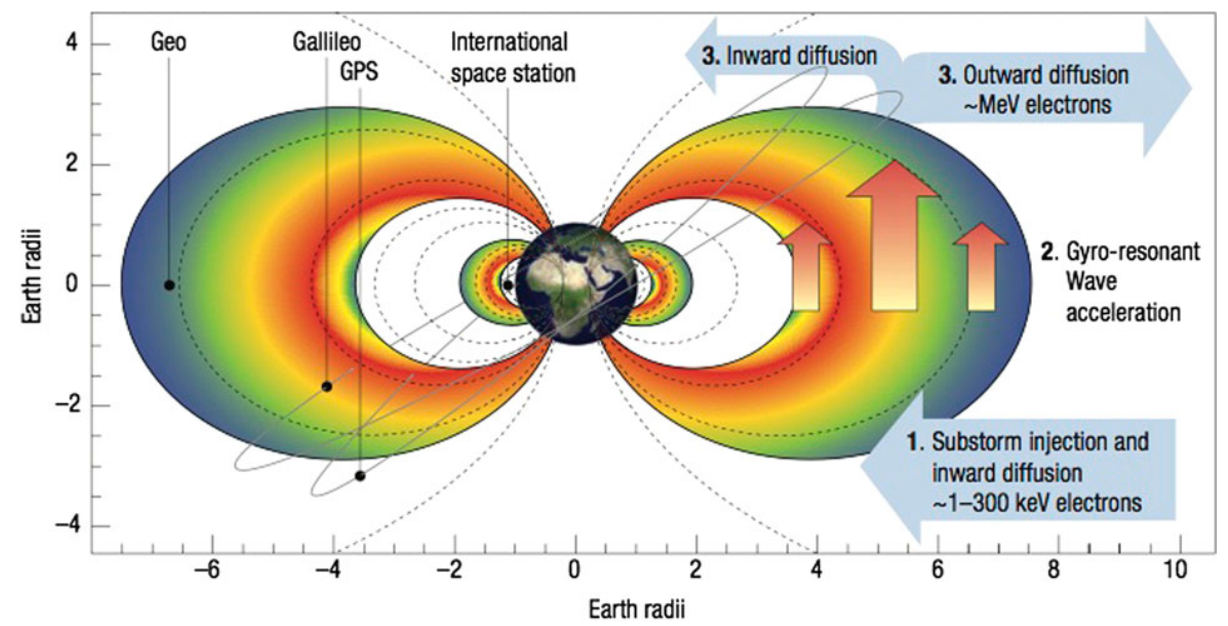

Fig. 7 Cartoon showing a possible scenario for the production of relativistic electrons in Earth's radiation belts. Reprinted by permission from Macmillan Publishers Ltd: Nature Physics (Horne 2007), copyright (2007) 


\subsection{Radial Transport}

Electric or magnetic field variations that occur on the timescale of a particle's gradientcurvature drift violate the third adiabatic invariant, driving the particle in the radial direction. Since the field variations are still slow relative to the gyration and bounce motion, the first and second adiabatic invariants are conserved. In particular, conservation of the first invariant (Eq. (3)) implies that the particle's perpendicular energy must increase as it moves to a region of stronger magnetic field (radially inward). For equatorially mirroring particles $(J=0)$, conservation of the second invariant (Eq. (5)) ensures that the parallel energy remains zero, thus one can easily see that the total energy of the particle increases in this case. This is essentially betatron acceleration; acceleration occurs when the particle drift motion is in phase (resonant) with variations in the induction electric field produced by the changing magnetic field. More generally, the particle pitch angle will increase with decreasing $L$ in order to conserve $\mu$ and $J$ (Schulz and Lanzerotti 1974), so the energy gained is less than that for equatorial particles where the energy gain is directly proportional to the ratio of initial and final magnetic field strengths. It is for this reason that radial transport is expected to lead to pancake-like pitch angle distributions (peaked near $90^{\circ}$ ).

Radial transport in the radiation belts occurs on two timescales relative to the drift motion, $\tau_{D}$, which is $\sim 10$ minutes at $\mathrm{MeV}$ energies (Fig. 3). Rapid radial transport $\left(\tau \leq \tau_{D}\right)$ moves particles across several L-shells on the drift timescale or faster. The first evidence of this occurring was during a large geomagnetic storm in March 1991. In this unusual event, electrons were rapidly (within minutes) transported all the way into $L=2-3$, producing a new radiation belt with energies peaking above $15 \mathrm{MeV}$ and possibly extending to $50 \mathrm{MeV}$ (Blake et al. 1992). The observed features were reproduced with a test particle simulation using a simple model of the electric field based on observations from the CRRES spacecraft (Li et al. 1993). The storm was caused by a Coronal Mass Ejection (CME) when the associated interplanetary shock encountered Earth's magnetic field, causing a rapid compression. The azimuthal induction electric field due to the compression propagated rapidly inward. Particles in drift-resonance, moving along with the wave, were swept inward, gaining energy through the betatron acceleration mechanism. Because of the long lifetimes of highly energetic electrons at $L \sim 3$, the new radiation belt persisted for more than 10 years. More recently, Kress et al. (2007) successfully modeled similar events (Fig. 8) during the "Halloween Storms" of October-November 2003 by following test particles in the fields computed from the Lyon-Feder-Mobarry (LFM) MHD code (Lyon et al. 2004). They point out that this type of event is not as rare as previously thought. Therefore such events have the potential for significant impacts on spacecraft systems, since particles can be accelerated to such high energies so quickly. However, they are rare enough that this mechanism probably doesn't account for the majority of relativistic electron enhancements.

Slow radial transport $\left(\tau \gg \tau_{D}\right)$ moves particles inward over a period of days or longer. Variations in Earth's magnetic field can be driven by both fluctuations in the solar wind dynamic pressure (e.g., Takahashi and Ukhorskiy 2007, 2008) and the Kelvin-Helmholz instability as the solar wind sweeps past the flanks of the magnetosphere (e.g., Claudepierre et al. 2008). These drive global Ultra Low Frequency (ULF) waves in the magnetosphere with $\mathrm{mHz}$ frequencies, comparable to the electron drift frequency. Such variations can again violate the third adiabatic invariant of trapped particles. Historically, the inward transport of electrons due to these variations has been treated as a diffusion process, satisfying the Fokker-Planck equation.

Radial diffusion was first suggested as an acceleration mechanism by Kellogg (1959) who considered the diffusion of solar electrons as a source for inner zone electrons. Parker 
Fig. 8 Test particle trajectory (black line) moving in the simulated azimuthal electric field (color bar), looking down onto the equatorial plane, following the October 29, 2003 interplanetary shock arrival. The electron shown was energized from 5 to $15 \mathrm{MeV}$ as it was transported inwards. Reproduced from Kress et al. (2007), copyright 2007, American Geophysical Union

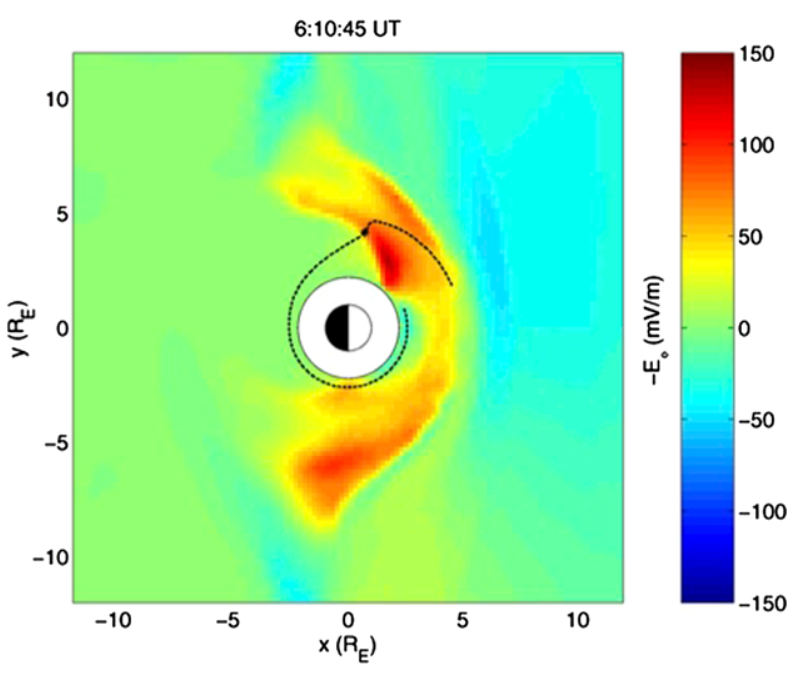

(1960) developed the diffusion theory, considering sudden compressions of the magnetic field that might occur, for example, upon arrival of an interplanetary shock. Davis and Chang (1962) first applied the Fokker-Planck equation rather than the heuristically derived diffusion equation of Parker, obtaining slightly different results. Fälthammar (1965) first considered the more general case of fluctuating magnetic and electric fields over a range of frequencies. The theory was further developed by many others; reviews of these early developments are given in e.g. Fälthammar (1968) and Tverskoy (1969).

A significant amount of work has been carried out to both model the effects of diffusive transport and to experimentally test whether the mechanism can reproduce observed features of the radiation belts. Lanzerotti et al. (1970) used data from Explorer 15 to estimate diffusion coefficients and electron lifetimes. They also provide a nice summary of results by other authors around that time in their Table 1. A textbook discussion of diffusion in the radiation belts and some early observational evidence can be found in Schulz and Lanzerotti (1974).

A multitude of computer simulations have been carried out over the last decade to further investigate radial diffusion. For example, Elkington et al. (1999) investigated the importance of toroidal-mode ULF waves and the convection electric field for equatorially mirroring particles. Shprits et al. (2005) considered a variable lifetime parameterized by geomagnetic activity and were able to reproduce the gross features observed by the CRRES satellite. See Shprits et al. (2008a) and references therein for a more comprehensive discussion of modeling results.

Observational tests of the radial diffusion mechanism have included analysis of both ULF wave data and energetic particle data. Several studies have found a correlation between ULF wave power and the rise in relativistic electron flux in the radiation belts (e.g., Baker et al. 1998; Rostoker et al. 1998; Hudson et al. 2000). The correlation does not necessarily imply causality, since both the ULF power and electron flux are correlated with increases in solar wind velocity, but it is suggestive.

Brautigam and Albert (2000) carried out a detailed study of one storm using data from CRRES and a radial diffusion model employing time dependent diffusion coefficient and outer boundary. They concluded that the observed increase in the high energy electron intensity was inconsistent with radial diffusion, and suggested energization by whistler-mode 
chorus waves as an alternative mechanism. Several studies have examined the radial gradient in the electron phase space density as a test for the radial diffusion mechanism (e.g., Green and Kivelson 2004; Iles et al. 2006; Chen et al. 2007). Because the energy increases as particles are transported inward, such studies require conversion of the measured particle intensities in fixed energy channels to phase space density at fixed values of the adiabatic invariants. Net inward diffusion of a source at large distances requires a radial gradient for fixed $\mu$ and $J$. However, these studies have generally found a peak in the phase space density near $L=4-5$, suggesting that the acceleration is occurring closer to Earth. Some uncertainty in these results is introduced because the calculation of the third invariant requires knowledge of the magnetic field around the entire particle drift orbit, thus these studies rely on models of the magnetic field. Additionally, other processes exist which may lead to peaks in phase space density (e.g., Degeling et al. 2008). Moreover, recent work showed that particle drift orbits are not closed for a range of equatorial pitch angles, even at geosynchronous orbit, implying that the third invariant is not defined for part of the population (Ukhorskiy et al. 2011a). Thus, examination of the phase space density radial gradient at or beyond geosynchronous orbit may be problematic.

Slow radial transport is not necessarily diffusive. Large deviations from diffusive transport can lead to spikes in phase space density due to phase correlations in the electron motion that do not have time to decay (Ukhorskiy et al. 2006a; Ukhorskiy and Sitnov 2008). This suggests another reason why peaks in phase space density may not provide a conclusive test of the radial diffusion mechanism. Such effects may also explain the very different outcomes for different magnetic storms that are otherwise similar (Reeves et al. 2003). These results imply that the behavior is only diffusive in an average sense, suggesting that the outcomes for a particular storm can only be predicted probabilistically.

Finally, one must consider whether radial transport alone, whether diffusive or not, is sufficient to account for the observed enhancements in the radiation belts. Fox et al. (2006) considered a source in the magnetotail beyond $10 R_{E}$ and determined that adiabatic radial transport, conserving the first and second invariants, could not explain the intensity of $>1 \mathrm{MeV}$ electrons observed at smaller radial distances $(L=5-7)$. This does not rule out the magnetotail as a source of radiation belt electrons, but does imply that further non-adiabatic acceleration is required.

More recently, Ukhorskiy et al. (2011a) investigated particle motion in the drift orbit bifurcation region, including three dimensional dynamic effects, and noted that these particles can be transported outward since the second adiabatic invariant is not conserved. An important feature of this outward radial transport is that it conserves the particle energy. Thus, one can envision a scenario in which particles that are transported inward, gaining energy through the betatron acceleration mechanism described above, are subsequently transported back out, conserving their recently gained energy. They can then be again transported inwards, gaining additional energy. Through this "recirculation" process, some particles were shown to gain a factor of two more energy than through radial transport alone. This work considered only a simple model for radial diffusion in a static magnetic field, and the importance of the process for the bulk of radiation belt particles in the real system is not yet clear. But, it does serve as a reminder that the particle dynamics in this region are quite complicated and can lead to new effects that must be considered. In particular, the dynamic nature of the magnetic field is likely to prove important in ways yet unknown.

Although radial transport probably does occur in the radiation belts, evidence now suggests that some other mechanism must also be acting to explain the rapid energization of electrons. The mechanism currently favored is acceleration through gyro-resonant interaction with plasma waves. 


\subsection{Wave-Particle Interactions}

Resonant wave-particle interactions can violate the first and second adiabatic invariants, causing a change in particle energy and/or pitch angle. A recent review of the quasi-linear approach to this problem, and the plasma waves relevant for radiation belt acceleration is given by Shprits et al. (2008b). Resonant interactions between a particle and a wave allow for exchange of energy and occur when the Doppler-shifted wave frequency matches the gyration of the particle:

$$
\omega-k_{\|} v_{\|}=\frac{n \Omega_{e}}{\gamma}
$$

where $\omega$ is the wave frequency, $k_{\|}$is the component of the wave vector parallel to the magnetic field, $v_{\|}$is the parallel particle velocity, $\Omega_{e}$ is the non-relativistic electron cyclotron frequency, $\gamma$ is the relativistic factor, and $n$ is a positive or negative integer corresponding to the order of the cyclotron resonance. The interactions of the particle with multiple waves is usually modeled as a diffusion in phase space, in accordance with quasi-linear theory. Energy diffusion and pitch angle diffusion are generally treated separately, though some work has been done to investigate the importance of cross-diffusion terms (Albert 2009). Diffusion coefficients are calculated using prescribed waves fields, typically based on statistical estimates of wave spectral power in the inner magnetosphere (e.g., Glauert and Horne 2005; Summers et al. 2007). These can then be used to numerically solve the Fokker-Planck equation. Most treatments have assumed parallel propagation of the waves, although obliquely-

Fig. 9 Example of very large amplitude whistler waves as measured by the Wind spacecraft. Reproduced from Wilson et al. (2011), copyright 2011, American Geophysical Union
TDSS: 11-13-1998/18:20:59,170 UT

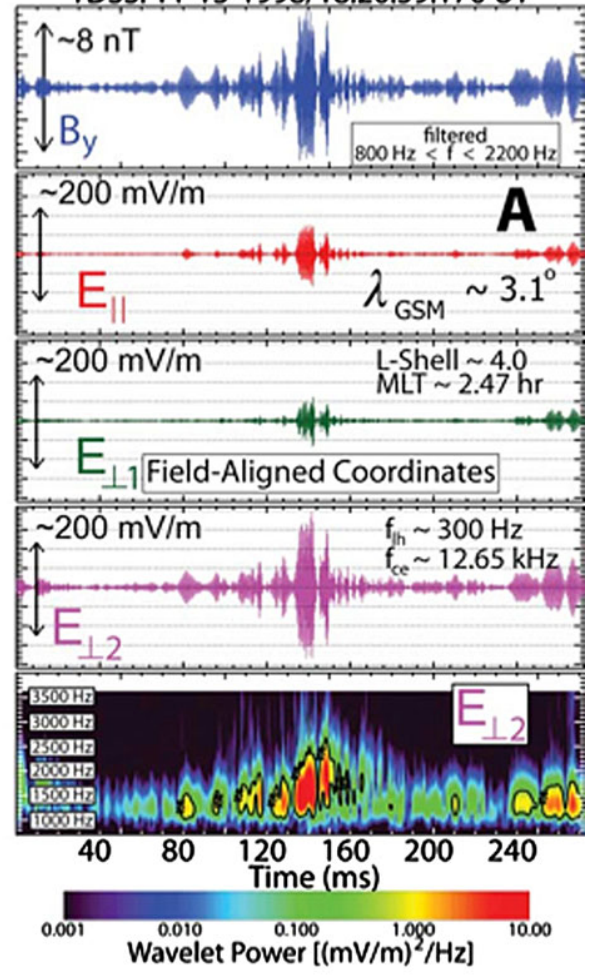


propagating whistler-mode waves are often observed off of the equator (e.g., Santolík et al. 2009; Agapitov et al. 2011; Li et al. 2011). Test particle simulations have been carried out to investigate the contribution of higher order resonances (Roth et al. 1999). Recent work by Artemyev et al. (2012) shows that the effects of higher-order resonances contribute significantly to the pitch angle diffusion coefficients in the case of nonparallel wave propagation.

Gyro-resonant interaction with whistler mode waves was first suggested as a loss mechanism for radiation belt particles, through pitch angle scattering into the atmospheric loss cone (e.g., Dungey 1963; Cornwall 1964; Kennel and Petschek 1966). However, it has since been shown that whistler-mode chorus (e.g., Horne and Thorne 1998; Summers et al. 1998) and magnetosonic waves (Horne et al. 2007) can lead to significant

Fig. 10 Energetic electron intensities versus energy and time for planets with strong magnetic fields as measured by ISEE (top panel) and Voyager (other panels). The intensity color scale is the same for each panel and the "M" on each panel represents a magnetopause crossing.

Reproduced from Mauk and Fox 2010), copyright 2010, American Geophysical Union
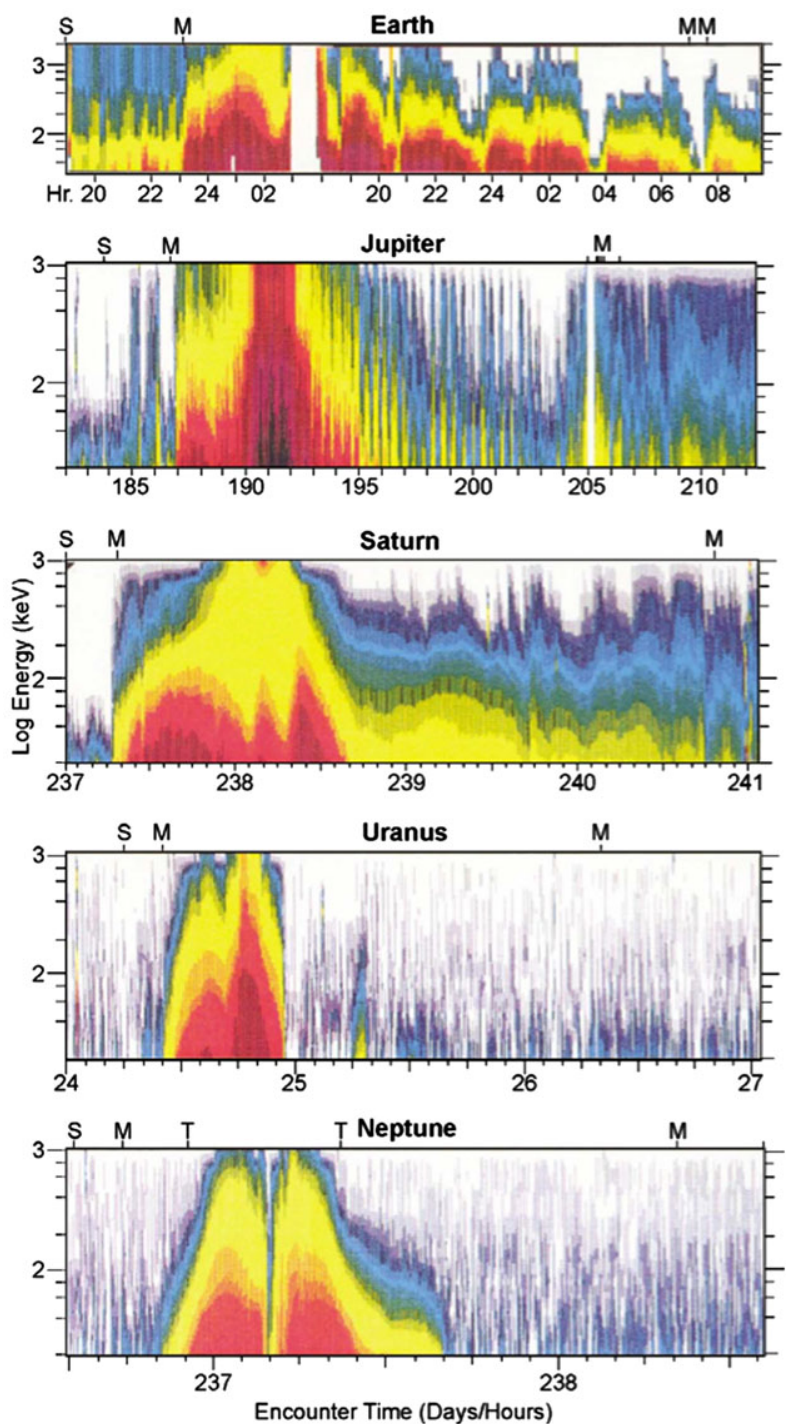
energization. As mentioned in the previous section, local scattering by wave-particle interactions provides one explanation for observed peaks in phase space density.

The recent discovery of very large amplitude whistler waves in the magnetosphere (Cattell et al. 2008) raises new questions about particle acceleration in the radiation belts. The wave amplitudes are so large (Fig. 9) that particles can gain significant energy during a single interaction with a wave. In this case, quasi-linear theory is not applicable and the wave-particle interaction can lead to non-linear phase trapping, first considered by Karpman et al. (1975) and more recently discussed by e.g., Bortnik et al. (2008), Shklyar and Matsumoto (2009), and Kellogg et al. (2010).

\section{Summary}

The radiation belts are a rich environment for investigating particle dynamics, with important applications to other astrophysical systems. It was recognized very early on that radiation belts might exist at other planets (Van Allen 1959c). We now know that all of the strongly magnetized planets in our solar system have radiation belts (Fig. 10). Earth's radiation belts are the most accessible and can be used to test the hypothesized acceleration mechanisms discussed in this review. Meanwhile, the JUNO mission is on its way to Jupiter with an expected arrival in 2016. Perhaps the next mission will visit Uranus to investigate particle acceleration in its radiation belts. The ability to do comparative studies provides us an opportunity to test our understanding for differing environments.

Although Earth's radiation belts are relatively accessible, there are many important questions so far unanswered. The radiation belts are a complex system in two respects. Particles trapped in this region are subjected to a multitude of different processes as they drift around the earth (Fig. 11). For example, they may interact with whistler-mode waves on the dayside and EMIC waves on the nightside, they may encounter the bifurcation region near noon and the stretched, highly curved field lines of the tail on the nightside. Large scale variations of the magnetic field, such as ULF waves or the build-up of the ring current, affect them in a global sense. The cumulative effect of these different mechanisms can rapidly move particles in phase space, and the interplay between different mechanisms cannot be ignored. On

Fig. 11 Schematic of important processes and mechanisms controlling the trapped population

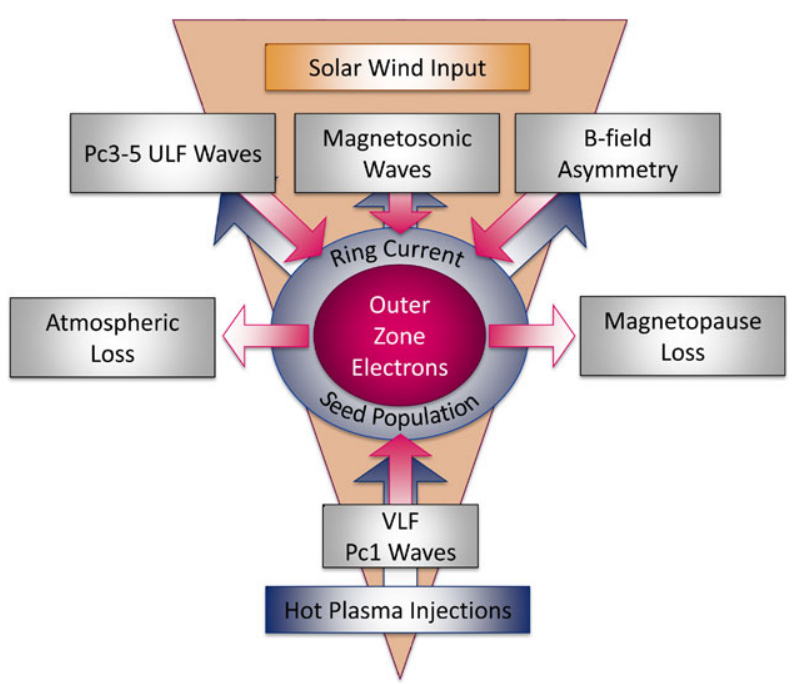


the other hand, even a single mechanism acting on the particles can be non-linear in terms of the response to a given input. It's not yet clear which of these is more important. We don't yet know if the variety of responses that we observe is due mainly to the interplay between mechanisms or the complexity of a single dominant mechanism. Or perhaps the dominant mechanism is quite simple, but the output response is complex because the driver (the solar wind) is itself complex.

These questions add a new dimension to our understanding and our approach for studying radiation belt physics. How do we search for the simple rules that give rise to the complex behavior? It may turn out that the system is not deterministic, but we may still find patterns that emerge and reveal the underlying physics.

Acknowledgements This work was supported by NSF ATM-0457561 at Dartmouth. The authors would also like to thank A.Y. Ukhorskiy for useful discussions and assistance with Fig. 11, and Michael Schulz for suggested references.

Open Access This article is distributed under the terms of the Creative Commons Attribution License which permits any use, distribution, and reproduction in any medium, provided the original author(s) and the source are credited.

\section{References}

B. Abel, R.M. Thorne, Electron scattering loss in Earth's inner magnetosphere, 1: dominant physical processes. J. Geophys. Res. 103, 2385 (1998)

O. Agapitov, V. Krasnoselskikh, Y.V. Khotyaintsev, G. Rolland, A statistical study of the propagation characteristics of whistler waves observed by cluster. Geophys. Res. Lett. 38(2), 20103 (2011)

J.M. Albert, The coupling of quasi-linear pitch angle and energy diffusion. J. Atmos. Sol.-Terr. Phys. 71(1), 1664-1668 (2009)

H. Alfvén, Theory of magnetic storms and of the Aurorae. K. Sven. Vetenskapsakad. Handl., Ser. 3 (1939)

S.V. Apatenkov, V.A. Sergeev, M.V. Kubyshkina, R. Nakamura, W. Baumjohann, A. Runov, I. Alexeev, A. Fazakerley, H. Frey, S. Muhlbachler, P.W. Daly, J.A. Sauvaud, N. Ganushkina, T. Pulkkinen, G.D. Reeves, Y. Khotyaintsev, Multi-spacecraft observation of plasma dipolarization/injection in the inner magnetosphere. Ann. Geophys. 25(3), 801-814 (2007)

A. Artemyev, O. Agapitov, H. Breuillard, V. Krasnoselskikh, G. Rolland, Electron pitch-angle diffusion in radiation belts: the effects of whistler wave oblique propagation (2012)

D.N. Baker, P.R. Higbie, R.D. Belian, E.W. Hones, Do Jovian electrons influence the terrestrial outer radiation zone. Geophys. Res. Lett. 6, 531-534 (1979a)

D.N. Baker, R.D. Belian, P.R. Higbie, E.W.J. Hones, High-energy magnetospheric protons and their dependence on geomagnetic and interplanetary conditions. J. Geophys. Res. 84, 7138-7154 (1979b)

D.N. Baker, E.W.J. Hones, P.R. Higbie, R.D. Belian, P. Stauning, Global properties of the magnetosphere during a substorm growth phase-a case study. J. Geophys. Res. 86, 8941-8956 (1981)

D.N. Baker, P.R. Higbie, T.A. Fritz, B. Wilken, W. Stuedemann, S.M. Kaye, M.G. Kivelson, T.E. Moore, A.J. Masley, P.H. Smith, Observation and modeling of energetic particles at synchronous orbit on July 29, 1977. J. Geophys. Res. 87, 5917-5932 (1982)

D.N. Baker, R.W. Klebesadel, P.R. Higbie, J.B. Blake, Highly relativistic electrons in the Earth's outer magnetosphere, I: lifetimes and temporal history 1979-1984. J. Geophys. Res. 91, 4265-4276 (1986)

D.N. Baker, R.L. McPherron, T.E. Cayton, R.W. Klebesadel, Linear prediction filter analysis of relativistic electron properties at 6.6 R(E). J. Geophys. Res. 95, 15133-15140 (1990)

D.N. Baker, S.G. Kanekal, M.D. Looper, J.B. Blake, R.A. Mewaldt, Jovian, solar, and other possible sources of radiation belt particles (1996)

D.N. Baker, X. Li, S.G. Kanekal, K.W. Ogilvie, R.P. Lepping, J.B. Blake, L.B. Callis, G. Rostoker, H.J. Singer, G.D. Reeves, A strong CME-related magnetic cloud interaction with the Earth's magnetosphere: ISTP observations of rapid relativistic electron acceleration on May 15, 1997. Geophys. Res. Lett. 25(1), 2975-2978 (1998)

D.N. Baker, S.G. Kanekal, X. Li, S.P. Monk, J. Goldstein, J.L. Burch, An extreme distortion of the Van Allen belt arising from the 'Hallowe'en' solar storm in 2003. Nature 432(7), 878-881 (2004)

D.N. Baker, S.G. Kanekal, R.B. Horne, N.P. Meredith, S.A. Glauert, Low-altitude measurements of 2-6 MeV electron trapping lifetimes at $1.5 \leq L \leq 2.5$. Geophys. Res. Lett. 34(2), 20110 (2007) 
J.B. Blake, W.A. Kolasinski, R.W. Fillius, E.G. Mullen, Injection of electrons and protons with energies of tens of MeV into $L$ less than 3 on 24 March 1991. Geophys. Res. Lett. 19, 821-824 (1992)

J. Bortnik, R.M. Thorne, U.S. Inan, Nonlinear interaction of energetic electrons with large amplitude chorus. Geophys. Res. Lett. 35(21), 21102 (2008)

D.H. Brautigam, J.M. Albert, Radial diffusion analysis of outer radiation belt electrons during the October 9 , 1990, magnetic storm. J. Geophys. Res. 105, 291 (2000)

C. Cattell, J.R. Wygant, K. Goetz, K. Kersten, P.J. Kellogg, T. von Rosenvinge, S.D. Bale, I. Roth, M. Temerin, M.K. Hudson, R.A. Mewaldt, M. Wiedenbeck, M. Maksimovic, R. Ergun, M. Acuna, C.T. Russell, Discovery of very large amplitude whistler-mode waves in Earth's radiation belts. Geophys. Res. Lett. 35, 01105 (2008)

Y. Chen, G.D. Reeves, R.H.W. Friedel, The energization of relativistic electrons in the outer Van Allen radiation belt. Nat. Phys. 3(9), 614-617 (2007)

N.C. Christofilos, The Argus experiment. J. Geophys. Res. 64, 869 (1959a)

N.C. Christofilos, The Argus experiment. Proc. Natl. Acad. Sci. USA 45, 1144 (1959b)

S.P. Christon, D.L. Chenette, D.N. Baker, D. Moses, Relativistic electrons at geosynchronous orbit, interplanetary electron flux, and the 13-month Jovian synodic year. Geophys. Res. Lett. 16, 1129-1132 (1989)

J.B. Cladis, L.F. Chase, W.L. Imhof, D.J. Knecht, Energy spectrum and angular distributions of electrons trapped in the geomagnetic field. J. Geophys. Res. 66, 2297 (1961)

S.G. Claudepierre, S.R. Elkington, M. Wiltberger, Solar wind driving of magnetospheric ULF waves: pulsations driven by velocity shear at the magnetopause. J. Geophys. Res. 113(A), 05218 (2008)

J.M. Cornwall, Scattering of energetic trapped electrons by very-low-frequency waves. J. Geophys. Res. 69, 1251 (1964)

J.A. Crawford, Fermi acceleration of electrons in the outer van Allen belt. Phys. Rev. Lett. 3, 316 (1959)

G.T. Davidson, P.C. Filbert, R.W. Nightingale, W.L. Imhof, J.B. Reagan, Observations of intense trapped electron fluxes at synchronous altitudes. J. Geophys. Res. 93, 77-95 (1988)

L.J. Davis, D.B. Chang, On the effect of geomagnetic fluctuations on trapped particles. J. Geophys. Res. 67, 2169-2179 (1962)

A.W. Degeling, L.G. Ozeke, R. Rankin, I.R. Mann, K. Kabin, Drift resonant generation of peaked relativistic electron distributions by Pc 5 ULF waves. J. Geophys. Res. 113(A), 02208 (2008)

A.J. Dessler, The Vernov radiation belt (almost). Science 226, 915 (1984)

S. Dubyagin, V. Sergeev, S. Apatenkov, V. Angelopoulos, A. Runov, R. Nakamura, W. Baumjohann, J. McFadden, D. Larson, Can flow bursts penetrate into the inner magnetosphere? Geophys. Res. Lett. 38(8), 08102 (2011)

J.W. Dungey, Loss of Van Allen electrons due to whistlers. Planet. Space Sci. 11, 591 (1963)

S.R. Elkington, M.K. Hudson, A.A. Chan, Acceleration of relativistic electrons via drift-resonant interaction with toroidal-mode Pc-5 ULF oscillations. Geophys. Res. Lett. 26, 3273 (1999)

S.R. Elkington, M. Wiltberger, A.A. Chan, D.N. Baker, Physical models of the geospace radiation environment. J. Atmos. Sol.-Terr. Phys. 66(1), 1371-1387 (2004)

H. Elliot, The Van Allen particles. Rep. Prog. Phys. 26, 145 (1963)

C.G. Fälthammar, Effects of time-dependent electric fields on geomagnetically trapped radiation. J. Geophys. Res. 70, 2503-2516 (1965)

C.G. Fälthammar, Radial diffusion by violation of the third adiabatic invariant, in Earth's Particles and Fields; Proceedings of the NATO Advanced Study Institute Held at Freising (1968), p. 157

T.A. Farley, M. Walt, Source and loss processes of protons of the inner radiation belt. J. Geophys. Res. 76, 8223-8240 (1971)

T.A. Farley, A.D. Tomassian, M. Walt, Source of high-energy protons in the Van Allen radiation belt. Phys. Rev. Lett. 25(1), 47-49 (1970)

N.J. Fox, B.H. Mauk, J.B. Blake, Role of non-adiabatic processes in the creation of the outer radiation belts. Geophys. Res. Lett. 33(1), 18108 (2006)

S.C. Freden, R.S. White, Particle fluxes in the inner radiation belt. J. Geophys. Res. 65, 1377 (1960)

S.C. Freden, R.S. White, Trapped proton and cosmic-ray albedo neutron fluxes. J. Geophys. Res. 67, 25 (1962)

S.A. Glauert, R.B. Horne, Calculation of pitch angle and energy diffusion coefficients with the PADIE code. J. Geophys. Res. 110(A), 04206 (2005)

T. Gold, Origin of the radiation near the Earth discovered by means of satellites. Nature 183(4658), 355 (1959)

J.C. Green, M.G. Kivelson, Relativistic electrons in the outer radiation belt: differentiating between acceleration mechanisms. J. Geophys. Res. 109(A), 03213 (2004)

N.L. Grigorov, M.A. Kondrat'eva, M.I. Panasiuk, C.A. Tret'iakova, J.H.J. Adams, J.B. Blake, M. Schulz, R.A. Mewaldt, A.J. Tylka, Evidence for trapped anomalous cosmic ray oxygen ions in the inner magnetosphere. Geophys. Res. Lett. 18, 1959-1962 (1991) 
W.N. Hess, G.D. Mead, M.P. Nakada, Advances in particles and field research in the satellite era. Rev. Geophys. Space Phys. 3, 521 (1965)

W.N. Hess, Van Allen belt protons from cosmic-ray neutron leakage. Phys. Rev. Lett. 3, 11 (1959)

W.N. Hess, Energetic particles in the inner Van Allen belt. Space Sci. Rev. 1, 278 (1962)

R.B. Horne, R.M. Thorne, Potential waves for relativistic electron scattering and stochastic acceleration during magnetic storms. Geophys. Res. Lett. 25, 3011 (1998)

R.B. Horne, R.M. Thorne, S.A. Glauert, N.P. Meredith, D. Pokhotelov, O. Santolik, Electron acceleration in the Van Allen radiation belts by fast magnetosonic waves. Geophys. Res. Lett. 34, 17107 (2007)

R.B. Horne, Plasma astrophysics: acceleration of killer electrons. Nat. Phys. 3(9), 590-591 (2007)

M.K. Hudson, S.R. Elkington, J.G. Lyon, C.C. Goodrich, Increase in relativistic electron flux in the inner magnetosphere: ULF wave mode structure. Adv. Space Res. 25(1), 2327-2337 (2000)

M.K. Hudson, B. Kress, H. Mueller, J. Zastrow, J. Bernardblake, Relationship of the Van Allen radiation belts to solar wind drivers. J. Atmos. Sol.-Terr. Phys. 70, 708 (2008)

R.H.A. Iles, N.P. Meredith, A.N. Fazakerley, R.B. Horne, Phase space density analysis of the outer radiation belt energetic electron dynamics. J. Geophys. Res. 111(A), 03204 (2006)

G. Kanbach, C. Reppin, V. Schoenfelder, Support for CRAND theory from measurements of earth albedo neutrons between 70 and $250 \mathrm{MeV}$. J. Geophys. Res. 79, 5159-5165 (1974)

S.G. Kanekal, D.N. Baker, J.B. Blake, Multisatellite measurements of relativistic electrons: global coherence. J. Geophys. Res. 106(A), 29721-29732 (2001)

S.G. Kanekal, R.H.W. Friedel, G.D. Reeves, D.N. Baker, J.B. Blake, Relativistic electron events in 2002: studies of pitch angle isotropization. J. Geophys. Res. 110(A), 12224 (2005)

V.I. Karpman, I.N. Istomin, D.R. Shkliar, Effects of nonlinear interaction of monochromatic waves with resonant particles in the inhomogeneous plasma, in Workshop on Electrodynamics of Nonequilibrium Plasmas, vol. 11 (1975), pp. 278-284

P.J. Kellogg, Possible explanation of the radiation observed by Van Allen at high altitudes in satellites. Nuovo Cimento 11, 48-66 (1959)

P.J. Kellogg, C.A. Cattell, K. Goetz, S.J. Monson, L.B. Wilson, Electron trapping and charge transport by large amplitude whistlers. Geophys. Res. Lett. 37, 20106 (2010)

C.F. Kennel, H.E. Petschek, Limit on stably trapped particle fluxes. J. Geophys. Res. 71, 1 (1966)

B.T. Kress, M.K. Hudson, M.D. Looper, J. Albert, J.G. Lyon, C.C. Goodrich, Global MHD test particle simulations of $>10 \mathrm{MeV}$ radiation belt electrons during storm sudden commencement. J. Geophys. Res. 112, 09215 (2007)

S.M. Krimigis, E.T. Sarris, T.P. Armstrong, Observations of Jovian electron events in the vicinity of earth. Geophys. Res. Lett. 2, 561-564 (1975)

L.J. Lanzerotti, C.G. Maclennan, M. Schulz, Radial diffusion of outer-zone electrons: an empirical approach to third-invariant violation. J. Geophys. Res. 75, 5351-5371 (1970)

A.M. Lenchek, S.F. Singer, Geomagnetically trapped protons from cosmic-ray albedo neutrons. J. Geophys. Res. 67, 1263 (1962)

W. Li, J. Bortnik, R.M. Thorne, V. Angelopoulos, Global distribution of wave amplitudes and wave normal angles of chorus waves using THEMIS wave observations. J. Geophys. Res. 116(A), 12205 (2011)

X. Li, I. Roth, M. Temerin, J.R. Wygant, M.K. Hudson, J.B. Blake, Simulation of the prompt energization and transport of radiation belt particles during the March 24, 1991 SSC. Geophys. Res. Lett. 20, 2423-2426 (1993)

X. Li, D.N. Baker, M. Temerin, D. Larson, R.P. Lin, G.D. Reeves, M. Looper, S.G. Kanekal, R.A. Mewaldt, Are energetic electrons in the solar wind the source of the outer radiation belt? Geophys. Res. Lett. 24(8), 923-926 (1997)

X. Li, D.N. Baker, S.G. Kanekal, M. Looper, M. Temerin, Long term measurements of radiation belts by SAMPEX and their variations. Geophys. Res. Lett. 28(2), 3827-3830 (2001)

R.P. Lin, Wind observations of superthermal electrons in the interplanetary medium. Space Sci. Rev. 86, 61-78 (1998)

K.R. Lorentzen, M.D. Looper, J.B. Blake, Relativistic electron microbursts during the GEM storms. Geophys. Res. Lett. 28(1), 2573-2576 (2001)

J.G. Lyon, J.A. Fedder, C.M. Mobarry, The Lyon-Fedder-Mobarry (lfm) global mhd magnetospheric simulation code. J. Atmos. Sol.-Terr. Phys. 66, 1333 (2004)

L.R. Lyons, R.M. Thorne, C.F. Kennel, Pitch-angle diffusion of radiation belt electrons within the plasmasphere. J. Geophys. Res. 77, 3455-3474 (1972)

L.R. Lyons, R.M. Thorne, Equilibrium structure of radiation belt electrons. J. Geophys. Res. 78, 2142-2149 (1973)

W.M. MacDonald, M. Walt, Diffusion of electrons in the Van Allen radiation belt, 2: particles with mirroring points at low altitude. J. Geophys. Res. 67, 5025-5033 (1962)

B.H. Mauk, N.J. Fox, Electron radiation belts of the solar system. J. Geophys. Res. 115(A), 12220 (2010) 
B.H. Mauk, D.G. Mitchell, R.W. McEntire, C.P. Paranicas, E.C. Roelof, D.J. Williams, S.M. Krimigis, A. Lagg, Energetic ion characteristics and neutral gas interactions in Jupiter's magnetosphere. J. Geophys. Res. (2004)

N.P. Meredith, R.B. Horne, D. Summers, R.M. Thorne, R.H.A. Iles, D. Heynderickx, R.R. Anderson, Evidence for acceleration of outer zone electrons to relativistic energies by whistler mode chorus. Ann. Geophys. 20(7), 967-979 (2002)

R.A. Mewaldt, R.S. Selesnick, J.R. Cummings, Anomalous cosmic rays: the principal source of high energy heavy ions in the radiation belts, in Radiation Belts: Models and Standards, ed. by J.F. Lemaire. Geophysical Monograph, vol. 97 (1996)

R.A. Mewaldt, E.C. Stone, R.E. Vogt, Observations of Jovian electrons at 1 AU. J. Geophys. Res. 81, 23972400 (1976)

R.M. Millan, R.M. Thorne, Review of radiation belt relativistic electron losses. J. Atmos. Sol.-Terr. Phys. 69(3), 362-377 (2007)

R.M. Millan, R.P. Lin, D.M. Smith, K.R. Lorentzen, M.P. McCarthy, X-ray observations of MeV electron precipitation with a balloon-borne germanium spectrometer. Geophys. Res. Lett. 29(2), 47 (2002)

T.G. Northrop, E. Teller, Stability of the adiabatic motion of charged particles in the Earth's field. Phys. Rev. 117(1), 215-225 (1960)

M.K. Öztürk, R.A. Wolf, Bifurcation of drift shells near the dayside magnetopause. J. Geophys. Res. 112, 07207 (2007). doi:10.1029/2006JA012102

E.N. Parker, Geomagnetic fluctuations and the form of the outer zone of the Van Allen radiation belt. J. Geophys. Res. 65, 3117 (1960)

G.A. Paulikas, J.B. Blake, Effects of the solar wind on magnetospheric dynamics-energetic electrons at the synchronous orbit, in Washington DC American Geophysical Union Geophysical Monograph Series, vol. 1 (1979), pp. 180-202

A.M. Preszler, S. Moon, R.S. White, Atmospheric neutrons. J. Geophys. Res. 81, 4715-4722 (1976)

G.D. Reeves, K.L. McAdams, R.H.W. Friedel, T.P. O'Brien, Acceleration and loss of relativistic electrons during geomagnetic storms. Geophys. Res. Lett. 30, 36 (2003)

G.D. Reeves, S.K. Morley, R.H.W. Friedel, M.G. Henderson, T.E. Cayton, G. Cunningham, J.B. Blake, R.A. Christensen, D. Thomsen, On the relationship between relativistic electron flux and solar wind velocity: Paulikas and Blake revisited. J. Geophys. Res. 116(A), 02213 (2011)

J.G. Roederer, Dynamics of Geomagnetically Trapped Radiation (Springer, New York, 1970)

J.G. Roederer, On the adiabatic motion of energetic particles in a model magnetosphere. J. Geophys. Res. 72, 981 (1967)

M.N. Rosenbluth, C.L. Longmire, Stability of plasmas confined by magnetic fields. Ann. Phys. 1, 120 (1957)

G. Rostoker, S. Skone, D.N. Baker, On the origin of relativistic electrons in the magnetosphere associated with some geomagnetic storms. Geophys. Res. Lett. 25(1), 3701-3704 (1998)

I. Roth, M. Temerin, M.K. Hudson, Resonant enhancement of relativistic electron fluxes during geomagnetically active periods. Ann. Geophys. 17(5), 631-638 (1999)

P. Rothwell, C.E. McIlwain, Magnetic storms and the Van Allen radiation belts-observations from satellite 1958e (explorer IV). J. Geophys. Res. 65, 799 (1960)

A. Runov, V. Angelopoulos, X.Z. Zhou, X.J. Zhang, S. Li, F. Plaschke, J. Bonnell, A THEMIS multicase study of dipolarization fronts in the magnetotail plasma sheet. J. Geophys. Res. 116(A), 05216 (2011)

O. Santolík, D.A. Gurnett, J.S. Pickett, J. Chum, N. Cornilleau-Wehrlin, Oblique propagation of whistler mode waves in the chorus source region. J. Geophys. Res. (2009)

M. Schulz, L.J. Lanzerotti, Particle Diffusion in the Radiation Belts (Springer, Germany, 1974)

M. Schulz, G.T. Davidson, Limiting energy spectrum of a saturated radiation belt. J. Geophys. Res. 93, 59-76 (1988)

R.S. Selesnick, Low-altitude distribution of radiation belt electrons. J. Geophys. Res. 109(A11), 1-11 (2004)

R.S. Selesnick, Source and loss rates of radiation belt relativistic electrons during magnetic storms. J. Geophys. Res. 111(A), 04210 (2006)

R.S. Selesnick, M.D. Looper, R.A. Mewaldt, A theoretical model of the inner proton radiation belt. Space Weather 5(4), 04003 (2007)

V.P. Shabansky, Some processes in the magnetosphere. Space Sci. Rev. 12(3), 299-418 (1971)

D. Shklyar, H. Matsumoto, Oblique whistler-mode waves in the inhomogeneous magnetospheric plasma: resonant interactions with energetic charged particles. Surv. Geophys. 30, 55-104 (2009)

Y.Y. Shprits, R.M. Thorne, G.D. Reeves, R. Friedel, Radial diffusion modeling with empirical lifetimes: comparison with CRRES observations. Ann. Geophys. 23, 1467-1471 (2005)

Y.Y. Shprits, S.R. Elkington, N.P. Meredith, D.A. Subbotin, Review of modeling of losses and sources of relativistic electrons in the outer radiation belt I: radial transport. J. Atmos. Sol.-Terr. Phys. 70, 16791693 (2008a) 
Y.Y. Shprits, D.A. Subbotin, N.P. Meredith, S.R. Elkington, Review of modeling of losses and sources of relativistic electrons in the outer radiation belt, II: local acceleration and loss. J. Atmos. Sol.-Terr. Phys. 70, 1694 (2008b)

S.F. Singer, "Radiation belt" and trapped cosmic-ray albedo. Phys. Rev. Lett. 1, 171 (1958)

S.F. Singer, Nature and origin of radiation belts. J. Phys. Soc. Jpn. 17, 187 (1962)

L. Spitzer, Equations of motion for an ideal plasma. Astrophys. J. 116, 299 (1952)

D.P. Stern, A brief history of magnetospheric physics during the space age. Rev. Geophys. 34, 1 (1996)

C. Størmer, The Polar Aurora (Clarendon, Oxford, 1955)

D.R. Summers, R.M. Thorne, F. Xiao, Relativistic theory of wave-particle resonant diffusion with application to electron acceleration in the magnetosphere. J. Geophys. Res. 103, 20487 (1998)

D. Summers, B. Ni, N.P. Meredith, Timescales for radiation belt electron acceleration and loss due to resonant wave-particle interactions, 1: theory. J. Geophys. Res. 112(A), 04206 (2007)

K. Takahashi, A.Y. Ukhorskiy, Solar wind control of Pc5 pulsation power at geosynchronous orbit. J. Geophys. Res. 112(A), 11205 (2007)

K. Takahashi, A.Y. Ukhorskiy, Timing analysis of the relationship between solar wind parameters and geosynchronous Pc5 amplitude. J. Geophys. Res. 113(A), 12204 (2008)

B.J. Teegarden, F.B. McDonald, J.H. Trainor, W.R. Webber, E.C. Roelof, Interplanetary MeV electrons of Jovian origin. J. Geophys. Res. 79, 3615-3622 (1974)

R.M. Thorne, Radiation belt dynamics: the importance of wave-particle interactions. Geophys. Res. Lett. 37, 22107 (2010)

N.A. Tsyganenko, M.I. Sitnov, Modeling the dynamics of the inner magnetosphere during strong geomagnetic storms. J. Geophys. Res. 110, 03208 (2005)

D.L. Turner, Y. Shprits, M. Hartinger, V. Angelopoulos, Explaining sudden losses of outer radiation belt electrons during geomagnetic storms (2012)

B.A. Tverskoy, Main mechanisms in the formation of the Earth's radiation belts. Rev. Geophys. Space Phys. 7, 219-231 (1969)

A.Y. Ukhorskiy, B.J. Anderson, K. Takahashi, N.A. Tsyganenko, Impact of ULF oscillations in solar wind dynamic pressure on the outer radiation belt electrons. Geophys. Res. Lett. 33(6), 06111 (2006a)

A.Y. Ukhorskiy, B.J. Anderson, P.C. Brandt, N.A. Tsyganenko, Storm-time evolution of the outer radiation belt: transport and losses. J. Geophys. Res. 111, 11 (2006b). doi:10.1029/ 2006JA011690

A.Y. Ukhorskiy, M.I. Sitnov, R.M. Millan, B.T. Kress, The role of drift orbit bifurcations in energization and loss of electrons in the outer radiation belt. J. Geophys. Res. 116(A), 09208 (2011a)

A.Y. Ukhorskiy, M.I. Sitnov, Radial transport in the outer radiation belt due to global magnetospheric compressions. J. Atmos. Sol.-Terr. Phys. 70(1), 1714-1726 (2008)

A.Y. Ukhorskiy, B.H. Mauk, N.J. Fox, D.G. Sibeck, J.M. Grebowsky, Radiation belt storm probes: resolving fundamental physics with practical consequences. J. Atmos. Sol.-Terr. Phys. 73(1), 1417-1424 (2011b)

A.L. Vampola, Natural variations in the geomagnetically trapped electron population, Technical Report TR0059(6260-20)-17, The Aerospace Corporation, 1971

A.L. Vampola, The ESA outer zone electron model update, in Environment Modelling for Space-Based Applications, vol. 392, (1996), p. 151

J.A. Van Allen, Geomagnetically-trapped corpuscular radiation. Proc. R. Soc. Lond. Ser. A, Math. Phys. Sci. 253(1275), 525-529 (1959a)

J.A. Van Allen, Radiation belts around the Earth. Sci. Am. 200, 39-47 (1959b)

J.A. Van Allen, The geomagnetically trapped corpuscular radiation. J. Geophys. Res. 64(11), 1683-1689 $(1959 \mathrm{c})$

J.A. Van Allen, L.A. Frank, Radiation around the Earth to a radial distance of 107,400 km. Nature 183, 430 (1959a)

J.A. Van Allen, L.A. Frank, Radiation measurements to 658,300 km with Pioneer IV. Nature 184, 219 (1959b)

J.A. Van Allen, C.E. McIlwain, G.H. Ludwig, Radiation observations with satellite 1958. J. Geophys. Res. 64, 271 (1959a)

J.A. Van Allen, C.E. McIlwain, G.H. Ludwig, Satellite observations of electrons artificially injected into the geomagnetic field. J. Geophys. Res. 64, 877 (1959b)

S.N. Vernov, N.L. Grigorov, I.P. Ivanenko, A.I. Lebedinskii, V.S. Murzin, A.E. Chudakov, Possible mechanism of production of "terrestrial corpuscular radiation" under the action of cosmic rays. Sov. Phys. Dokl. 4, 154 (1959a)

S.N. Vernov, A.E. Chudakov, E.V. Gorchakov, J.L. Logachev, P.V. Vakulov, Study of the cosmic ray soft component by the 3rd soviet Earth satellite. Planet. Space Sci. 1, 86-93 (1959b)

J.I. Vette, The AE-8 trapped electron model environment, Technical Report NSSDC/WDC-A-RS 91-24, National Space Science Data Center, 1991 
M. Walt, W.M. MacDonald, Diffusion of electrons in the Van Allen radiation belt, 1: treatment of particles with mirroring points at high altitude. J. Geophys. Res. 67, 5013-5024 (1962)

H.I.J. West, R.M. Buck, J.R. Walton, Electron pitch-angle distributions throughout the magnetosphere as observed on OGO 5. J. Geophys. Res. 78, 1064 (1973)

D.J. Williams, J.F. Arens, L.J. Lanzerotti, Observations of trapped electrons at low and high altitudes. J. Geophys. Res. 73, 5673 (1968)

L.B.I. Wilson, C.A. Cattell, P.J. Kellogg, J.R. Wygant, K. Goetz, A. Breneman, K. Kersten, The properties of large amplitude whistler mode waves in the magnetosphere: propagation and relationship with geomagnetic activity. Geophys. Res. Lett. 38(1), 17107 (2011) 